\title{
TWISTED GRADED ALGEBRAS AND EQUIVALENCES OF GRADED CATEGORIES
}

\author{
J. J. ZHANG \\ [Received 28 September 1992-Revised 1 July 1993 and 10 October 1994]
}

\begin{abstract}
Let $A=\bigoplus_{n \geqslant 0} A_{n}$ be a connected graded $k$-algebra and let Gr-A denote the category of graded right $A$-modules with morphisms being graded homomorphisms of degree 0 . If $\left\{\tau_{n} \mid n \in \mathbb{Z}\right\}$ is a set of graded $k$-linear bijections of degree 0 from $A$ to itself satisfying

$$
\tau_{n}\left(y \tau_{m}(z)\right)=\tau_{n}(y) \tau_{n+m}(z)
$$

for all $l, m, n \in \mathbb{Z}$ and all $y \in A_{m}, z \in A_{l}$, we define a new graded associative multiplication * on the underlying graded $k$-vector space $\bigoplus_{n \geqslant 0} A_{n}$ by $y * z=y \tau_{m}(z)$ for all $y \in A_{m}, z \in A_{l}$. The graded algebra with the new multiplication $*$ is called a twisted algebra of $A$.
\end{abstract}

THEOREM. Let $A$ and $B$ be two connected graded algebras generated in degree 1 . Then the categories $\mathrm{Gr}-A$ and $\mathrm{Gr}-B$ are equivalent if and only if $A$ is isomorphic to a twisted algebra of $B$.

If algebras are noetherian, then Gelfand-Kirillov dimension, global dimension, injective dimension, Krull dimension, and uniform dimension are preserved under twisting. Moreover, we prove the following:

THEOREM. The following properties are preserved under twisting for connected graded noetherian algebras:

(a) Artin-Schelter Gorenstein (or Artin-Schelter regular);

(b) Auslander Gorenstein (or Auslander regular) and Cohen-Macaulay.

Some of these results are also generalized to certain semigroup-graded algebras.

\section{Introduction}

Let $k$ be a commutative ring and let $A=\bigoplus_{n \in \mathbb{Z}} A_{n}$ be a $\mathbb{Z}$-graded $k$-algebra. We denote by $\mathrm{Gr}-A$ the category of graded right $A$-modules, with morphisms being graded homomorphisms of degree 0 . Let $\tau=\left\{\tau_{n} \mid n \in \mathbb{Z}\right\}$ be a set of graded $k$-linear automorphisms of $A$. Then $\tau$ is called a twisting system if

$$
\tau_{n}\left(y \tau_{m}(z)\right)=\tau_{n}(y) \tau_{n+m}(z)
$$

for all $n, m, l \in \mathbb{Z}$ and $y \in A_{m}, z \in A_{l}$. If $f$ is a graded algebra automorphism of $A$, then the set $\left\{\tau_{n}:=f^{n} \mid n \in \mathbb{Z}\right\}$ satisfies (1.0.1) because $f^{n} \cdot f^{m}=f^{n+m}$. Hence it is a twisting system. In this sense, we can regard a twisting system as a generalized form of a graded algebra automorphism. Given a twisting system of $A$, say $\left\{\tau_{n} \mid n \in \mathbb{Z}\right\}$, we can define a new graded multiplication $*$ on the underlying graded $k$-module $\bigoplus_{n \in \mathbb{Z}} A_{n}$ by

$$
y * z=y \tau_{m}(z)
$$

for all $y \in A_{m}, z \in A_{l}$. The associativity of $*$ follows easily from (1.0.1). The new graded algebra $\left(\bigoplus_{n \in \mathbb{Z}} A_{n}^{*}, *\right)$ is called a twisted algebra of $A$. We prove the following:

THEOREM 1.1. If $A$ is isomorphic to a twisted algebra of $B$, then the categories $\mathrm{Gr}-A$ and $\mathrm{Gr}-B$ are isomorphic. 
A special case of Theorem 1.1 , when the twisting system is $\left\{\tau_{n}:=f^{n} \mid n \in \mathbb{Z}\right\}$ for a graded automorphism $f$ of $A$, is given by [3, Corollary 8.5]. A converse statement of Theorem 1.1 holds for connected graded rings. Recall that a graded algebra $A=\bigoplus_{n \in \mathbb{Z}} A_{n}$ is called connected graded if $A_{0}=k$ and $A_{n}=0$ for all $n<0$.

THEOREM 1.2. Let $k$ be a field and let $A$ and $B$ be connected graded $k$-algebras with $A_{1} \neq 0$. Then $A$ is isomorphic to a twisted algebra of $B$ if and only if Gr- $A$ is equivalent to $\mathrm{Gr}-B$.

Studying equivalences of categories of modules surely reminds us of the Morita theorems $[8, \S 3.12]$. One version of the Morita theorems also holds in the context of $\mathbb{Z}$-graded algebras. Let $\mathscr{C}_{A}$ denote the category of graded right $A$-modules, with morphisms being all graded homomorphisms (not only graded homomorphisms of degree zero). The category $\mathscr{C}_{A}$ is not abelian because the sum of two homomorphisms of different degrees is no longer graded, but it has most other properties of an abelian category. Similar to the Morita theorems, one can prove that $\mathscr{C}_{A}$ is equivalent to $\mathscr{C}_{B}$ via a functor which preserves the degree of homomorphisms if and only if $A \cong \underline{\operatorname{End}}\left(P_{B}\right)=\bigoplus_{n \in \mathbb{Z}} \operatorname{Hom}\left(P_{B}, P_{B}\right)_{n}$ for some graded right progenerator $P_{B}$. If this is the case, we call $\mathscr{C}_{A}$ and $\mathscr{C}_{B}$ (or $A$ and $B$ ) graded Morita equivalent (for related results see [7, Theorem 5.4] and [5]). It is easy to see that connected graded algebras $A$ and $B$ are graded Morita equivalent if and only if $A$ and $B$ are isomorphic. However, when we deal with graded algebras, it is more natural to consider the category $\mathrm{Gr}-A$, in which the morphisms are graded homomorphisms of degree 0 . If $\mathscr{C}_{A}$ and $\mathscr{C}_{B}$ are graded Morita equivalent, since $\mathrm{Gr}-A$ is a subcategory of $\mathscr{C}_{A}$, it is easy to prove that $\mathrm{Gr}-A$ and $\mathrm{Gr}-B$ are equivalent. The converse is not true, because we can construct a twisted algebra of $A$, say $B$, which is not isomorphic to $A$. For example, let $A$ be the polynomial ring $k[x, y]$ with $\operatorname{deg}(x)=\operatorname{deg}(y)=1$ and let $f$ be the automorphism defined by $f(x)=x$ and $f(y)=q y$ for some non-zero scalar $q$. Then $\left\{\tau_{n}:=f^{n} \mid n \in \mathbb{Z}\right\}$ is a twisting system of $k[x, y]$, and the twisted algebra is isomorphic to the skew polynomial ring $k_{q}[x, y], k\langle x, y\rangle /(x y-q y x)$. Similarly, if the automorphism is defined by $f(x)=x$ and $f(y)=y-x$, the twisted algebra is isomorphic to the Jordan polynomial algebra $k_{J}[x, y]=k\langle x, y\rangle /\left(x^{2}+x y-y x\right)$ (see Example 3.6). The algebras $k[x, y], k_{q}[x, y]$ (for $q \neq 1$ ), and $k_{J}[x, y]$ are not graded Morita equivalent, but, by Theorem 1.1, the categories Gr-k[x,y], $\mathrm{Gr}-k_{q}[x, y]$ and $\mathrm{Gr}-k_{J}[x, y]$ are isomorphic.

Recall that a property on rings and/or modules is called Morita invariant if it is preserved under Morita equivalences. A property $P$ is said to be a twisting invariant if, whenever a graded algebra $A$ has property $P$, then every twisted algebra of $A$ also has the property.

THEOREM 1.3. The following properties are twisting invariant for connected graded, finitely generated algebras:

(a) having Gelfand-Kirillov dimension $d_{1}$;

(b) having global dimension $d_{2}$;

(c) having Krull dimension $d_{3}$;

(d) having uniform dimension $d_{4}$; 
(e) being a domain;

(f) being noetherian;

(g) being semiprime graded Goldie.

For noetherian connected graded algebras, the following are twisting invariant:

(h) having injective dimension $d_{5}$;

(i) being Artin-Schelter Gorenstein (or Artin-Schelter regular);

(j) being Auslander Gorenstein (or Auslander regular) and Cohen-Macaulay.

On the other hand, some Morita invariants, such as being prime and being a PI ring, are not twisting invariants.

Some ideas in this paper are from non-commutative projective geometry [4]. Recall that QGr- $A$ is the quotient category $\mathrm{Gr}-A$ /Tors where Tors is the full subcategory of $\mathrm{Gr}-A$ consisting of all torsion $A$-modules. For a right $A$-module $M$, the image of $M$ in $\mathrm{QGr}-A$ is denoted by $M$. The degree shift of graded modules induces a shift operator $s$ of QGr- $A$. Following the notation of [4], we denote $\bigoplus_{n \in \mathbb{Z}} \operatorname{Hom}_{\mathrm{QGr}-A}\left(\mathscr{A}, s^{n}(\mathscr{A})\right)$ by $\Gamma(\mathscr{A})$.

THEOREM 1.4. Let $A$ and $B$ be $\mathbb{N}$-graded right noetherian.

1. If $A$ is isomorphic to a twisted algebra of $B$, then there is an equivalence functor $\mathscr{F}$ from QGr-A to QGr-B such that $\mathscr{F}\left(s^{n}(\mathscr{A})\right) \cong s^{n}(\mathscr{B})$ for all $n \in \mathbb{Z}$.

2. If there is an equivalence functor $\mathscr{F}$ from QGr-A to $\mathrm{QGr}-B$ such that $\mathscr{F}\left(s^{n}(\mathscr{A})\right) \cong s^{n}(\mathscr{B})$ for all $n \in \mathbb{Z}$, then $\Gamma(\mathscr{A})$ is isomorphic to a twisted algebra of $\Gamma(\mathscr{B})$.

The theory of twisting system and twisted algebra works for all semigroupgraded algebras, and Theorem 1.1 and a version of Theorem 1.2 are proved in general. For completeness, we also briefly introduce a twisted coalgebra of a graded coalgebra and a left-hand version of a twisted algebra which corresponds to equivalence of categories of graded left modules.

\section{Twisting systems and twisted algebras}

In this section we give the definitions of twisting system and twisted algebra and investigate their elementary properties.

Throughout the paper $\mathbb{G}$ will denote a (not necessarily commutative) semigroup, and $e$ will denote the unit of $\mathbb{G}$ unless indicated otherwise. A $k$-algebra $A$ is called $\mathbb{G}$-graded if

(a) $A=\bigoplus_{g \in \mathbb{G}} A_{g}$ where each $A_{g}$ is a $k$-submodule of $A$,

(b) $A_{g} A_{h} \subseteq A_{g h}$ for all $g, h \in \mathbb{G}$, and

(c) the identity of $A$ is in $A_{e}$.

A right $A$-module $M$ is called $\mathbb{G}$-graded if

(a) $M=\bigoplus_{g \in \mathbb{G}} M_{g}$ where each $M_{g}$ is a $k$-submodule of $M$, and

(b) $M_{g} A_{h} \subseteq M_{g h}$ for all $g, h \in \mathbb{G}$.

Let $M$ and $N$ be two $G$-graded modules. A $k$-linear map $f$ from $M$ to $N$ is called graded if $f\left(M_{g}\right) \subseteq N_{g}$ for all $g \in \mathbb{G}$. If, moreover, it is $A$-linear, then it is called a 
graded homomorphism. For simplicity, we usually omit prefixes $\mathbb{G}$ and/or $k$ if no confusion occurs. The basic facts about graded algebras and modules may be found in [13].

Note that a graded $k$-linear automorphism of $A$ is not necessarily an algebra automorphism. We give the following definition.

Definition 2.1. Let $A=\bigoplus_{g} A_{g}$ be a $\mathbb{G}$-graded $k$-algebra. A set of graded $k$-linear automorphisms of $A$, say $\tau=\left\{\tau_{g} \mid g \in \mathbb{G}\right\}$, is called a twisting system of $A$ if

$$
\tau_{g}\left(y \tau_{h}(z)\right)=\tau_{g}(y) \tau_{g h}(z)
$$

for all $g, h, l \in \mathbb{G}$ and all $y \in A_{h}, z \in A_{l}$.

Let $\operatorname{Aut}(A)$ denote the group of graded algebra automorphisms of $A$ and let $g \mapsto \tau_{g}$ be a semigroup homomorphism from $\mathbb{G}$ to $\operatorname{Aut}(A)$. Then $\tau_{g} \tau_{h}=\tau_{g h}$ for all $g, h \in \mathbb{G}$. Since each $\tau_{g}$ is a graded algebra automorphism of $A$, we obtain

$$
\tau_{g}\left(y \tau_{h}(z)\right)=\tau_{g}(y) \tau_{g} \tau_{h}(z)=\tau_{g}(y) \tau_{g h}(z)
$$

for all $g, h, l \in \mathbb{G}$ and $y \in A_{h}, z \in A_{l}$. Hence $\left\{\tau_{g} \mid g \in \mathbb{G}\right\}$ is a twisting system. If $\mathbb{G}=\mathbb{Z}$ and $f$ is a graded algebra automorphism of $A$, then the map $n \mapsto f^{n}$ is a group homomorphism from $\mathbb{Z}$ to $\operatorname{Aut}(A)$ and $\left\{f^{n} \mid n \in \mathbb{Z}\right\}$ is a twisting system of $A$. This also holds for $\mathbb{G}=\mathbb{N}$. A twisting system $\left\{\tau_{g} \mid g \in \mathbb{G}\right\}$ of $A$ is called algebraic if the map $g \mapsto \tau_{g}$ is a semigroup homomorphism from $\mathbb{G}$ to Aut $(A)$. The following is a trivial example in which each $\tau_{g}$ is not an algebra automorphism. Let $a$ be an invertible element of degree $e$. Define $\tau_{g}(y)=a y$ for all $y \in A$ and for all $g \in \mathbb{G}$. Then $\tau_{g}\left(y \tau_{h}(z)\right)=$ ayaz $=\tau_{g}(y) \tau_{g h}(z)$. Hence $\left\{\tau_{g} \mid g \in \mathbb{G}\right\}$ is a twisting system.

For convenience, we write down three identities which are all equivalent to (2.1.1). We will use these frequently later. For all $y \in A_{h}, z \in A_{l}$,

$$
\begin{gathered}
\tau_{g}(y z)=\tau_{g}(y) \tau_{g h} \tau_{h}^{-1}(z), \\
\tau_{g}^{-1}(y z)=\tau_{g}^{-1}(y) \tau_{h} \tau_{g h}^{-1}(z), \\
\tau_{g}^{-1}\left(y \tau_{g h}(z)\right)=\tau_{g}^{-1}(y) \tau_{h}(z) .
\end{gathered}
$$

Replacing $z$ by $\tau_{h}^{-1}(z)$ in (2.1.1), we see that (2.1.1) is equivalent to (2.1.2). Replacing $z$ by $\tau_{g h}(z)$ in (2.1.3), we see that (2.1.3) is equivalent to (2.1.4). Replacing $y$ by $\tau_{g}^{-1}(y)$ and applying $\tau_{g}$ to both sides of (2.1.4), we see that (2.1.1) is equivalent to (2.1.4).

Proposition 2.2. Let $\left\{\tau_{g} \mid g \in \mathbb{G}\right\}$ be a twisting system of $A$.

1. For every homogeneous element $a$ and for every $g \in G, \tau_{g}(a)$ is invertible if and only if $a$ is invertible.

2. For every $g \in G, \tau_{g}^{-1}(1)=\tau_{e}^{-1}(1)$, and $\tau_{e}^{-1}(1)$ is the inverse of $\tau_{e}(1)$, where 1 is the identity of $A$.

3. For every $y \in A, \tau_{e}(y)=\tau_{e}(1) y$ and $y=\tau_{e}^{-1}(1) \tau_{e}(y)$.

As a consequence, if $\tau_{e}(1)=1$, then $\tau_{g}(1)=1$ for all $g \in \mathbb{G}$ and $\tau_{e}(y)=y$ for all $y \in A$. 
Proof. 1. Let $h=\operatorname{deg}(a)$. By (2.1.2), $\tau_{g}(a z)=\tau_{g}(a) z_{1}$ where $z_{1}=\tau_{g h} \tau_{h}^{-1}(z)$. From this equality, we see that

(i) $a$ is right regular, that is, $a z \neq 0$ for any non-zero element $z$, if and only if $\tau_{g}(a)$ is right regular, and that

(ii) $a A=A$ if and only if $\tau_{g}(a) A=A$ because $\tau_{g}$ is a graded bijection and $\tau_{g}(a A)=\tau_{g}(a) A$.

As an easy fact, $a$ is invertible if and only if both $a$ is right regular and $a A=A$. Therefore $a$ is invertible if and only if $\tau_{g}(a)$ is invertible.

2. By (2.1.3),

$$
1=\tau_{g}^{-1}\left(\tau_{g}(1)\right)=\tau_{g}^{-1}\left(1 \cdot \tau_{g}(1)\right)=\tau_{g}^{-1}(1) \tau_{e}\left(\tau_{g e}^{-1}\left(\tau_{g}(1)\right)\right)=\tau_{g}^{-1}(1) \tau_{e}(1) .
$$

Hence $\tau_{g}^{-1}(1)$ is the inverse of $\tau_{e}(1)$ for all $g \in \mathbb{G}$. Consequently, $\tau_{g}^{-1}(1)=\tau_{e}^{-1}(1)$.

3. If $y$ is homogeneous, by (2.1.1),

$$
\tau_{e}(y)=\tau_{e}\left(1 \cdot \tau_{e} \tau_{e}^{-1}(y)\right)=\tau_{e}(1) \tau_{e \cdot e}\left(\tau_{e}^{-1}(y)\right)=\tau_{e}(1) y .
$$

Since $\tau_{e}$ is $k$-linear, the above is true for all $y$. By Part $2, \tau_{e}^{-1}(1)$ is the inverse of $\tau_{e}(1)$, so $y=\tau_{e}^{-1}(1) \tau_{e}(y)$.

Finally, we suppose that $\tau_{e}(1)=1$. By Part $2, \tau_{g}^{-1}(1)=\tau_{e}^{-1}(1)=1$, so $\tau_{g}(1)=1$. By Part 3, $\tau_{e}(y)=\tau_{e}(1) y=1 y=y$.

Let $\tau=\left\{\tau_{g} \mid g \in \mathbb{G}\right\}$ be a twisting system of a graded algebra $A$. We write $1_{\tau}=\tau_{e}^{-1}(1)$. By the above proposition, $\tau_{g}^{-1}(1)=1_{\tau}$ for all $g \in \mathbb{G}$. Hence $\tau_{g}\left(1_{\tau}\right)=1$. Note that there are examples in which $\tau_{g}(1) \neq \tau_{e}(1)$.

Proposition and Definition 2.3. Let $A$ be a graded algebra, and let $\tau=$ $\left\{\tau_{g} \mid g \in \mathbb{G}\right\}$ be a twisting system of $A$. Then there is a new graded and associative multiplication * on the underlying graded $k$-module $\oplus_{g} A_{g}$ defined by

$$
y * z=y \tau_{h}(z)
$$

for all $y \in A_{h}, z \in A_{l}$. The element $1_{\tau}$ is the identity with respect to $*$. The graded algebra $\left(\bigoplus_{g} A_{g}, *, 1_{\tau}\right)$ is called the twisted algebra of $A$ by $\tau$ and it is denoted by $A^{\tau}$.

Proof. By definition, * is a $k$-bilinear map from $A_{h} \otimes_{k} A_{l}$ to $A_{h l}$. For every $x \in A_{g}, y \in A_{h}$ and $z \in A_{l}$, we have

$$
(x * y) * z=\left(x \tau_{g}(y)\right) \tau_{g h}(z)=x\left(\tau_{g}(y) \tau_{g h}(z)\right)=x \tau_{g}\left(y \tau_{h}(z)\right)=x *(y * z) .
$$

Hence the multiplication $*$ is associative. By the definition of $*$ and Proposition 2.2.3,

$$
1_{\tau} * y=\tau_{e}^{-1}(1) \tau_{e}(y)=y
$$

and

$$
y * 1_{\tau}=y \tau_{h} \tau_{e}^{-1}(1)=y 1=y .
$$

Hence $1_{\tau}$ is the identity with respect to the new multiplication *. Therefore $\left(\bigoplus_{g} A_{g}, *, 1_{\tau}\right)$ is a $\mathbb{G}$-graded $k$-algebra.

Proposition 2.4. Let $A$ be a graded algebra. For every twisting system 
$\tau=\left\{\tau_{g} \mid g \in \mathbb{G}\right\}$ of $A$, there is another twisting system $\tau^{\prime}=\left\{\tau_{g}^{\prime} \mid g \in \mathbb{G}\right\}$ such that $\tau_{e}^{\prime}(1)=1$ and $A^{\tau} \cong A^{\tau^{\prime}}$.

Proof. Fix an element $s \in \mathbb{G}$ and let $\tau_{g}^{\prime}$ be $\tau_{s g} \tau_{s}^{-1}$ for all $g \in \mathbb{G}$. By Proposition 2.2.2, $\tau_{e}^{\prime}(1)=1$. We claim that $\tau^{\prime}:=\left\{\tau_{g}^{\prime} \mid g \in \mathbb{G}\right\}$ is a twisting system and that $\tau_{s}$ is a graded algebra isomorphism from $A^{\tau}$ to $A^{\tau^{\prime}}$. For every $y \in A_{h}, h \in A_{l}$, by (2.1.4) and (2.1.1), we have

$$
\tau_{s g} \tau_{s}^{-1}\left(y \tau_{s h} \tau_{s}^{-1}(z)\right)=\tau_{s g}\left(\tau_{s}^{-1}(y) \tau_{h} \tau_{s}^{-1}(z)\right)=\tau_{s g} \tau_{s}^{-1}(y) \tau_{s g h} \tau_{s}^{-1}(z)
$$

Hence $\tau^{\prime}=\left\{\tau_{g}^{\prime} \mid g \in \mathbb{G}\right\}$ is a twisting system of $A$. Let $*^{\prime}$ denote the multiplication of $A^{\tau^{\prime}}$. For every $y \in A_{h}, z \in A_{l}$,

$$
\tau_{s}(y * z)=\tau_{s}\left(y \tau_{h}(z)\right)=\tau_{s}(y) \tau_{s h}(z)=\tau_{s}(y) \tau_{s h} \tau_{s}^{-1}\left(\tau_{s}(z)\right)=\tau_{s}(y) *^{\prime} \tau_{s}(z) .
$$

Hence $\tau_{s}$ is a graded algebra isomorphism from $A^{\tau}$ to $A^{\tau^{\prime}}$.

By the above proposition, we may assume that $\tau_{e}(1)=1$ when we study a twisted algebra $A^{\tau}$. If $\tau_{e}(1)=1$, by Proposition $2.2, \tau_{e}$ is the identity map of $A$ and the identity of the twisted algebra $A^{\tau}$ is equal to the identity of $A$. Hence for all $a, b \in A_{e}, a * b=a \tau_{e}(b)=a b$. Therefore the subring $A_{e}$ of $A$ is equal to the subring $\left(A^{\tau}\right)_{e}$ of $A^{\tau}$. In particular, if $A=A_{e}$ then every twisted algebra of $A$ is $A$ itself.

By definition, $A$ and $A^{\tau}$ have the same underlying graded $k$-module $\bigoplus_{g} A_{g}$. The following proposition shows that we can define an equivalence relation, by using twisting systems, on the set of associative graded multiplications defined on $\bigoplus_{g} A_{g}$.

Proposition 2.5. Let $A$ be a graded algebra. Then the following statements hold.

1. (Reflexivity) $A$ is a twisted algebra of itself.

2. (Symmetry) if $B$ is a twisted algebra of $A$, then $A$ is a twisted algebra of $B$.

3. (Transitivity) if $B$ is a twisted algebra of $A$ and $C$ is a twisted algebra of $B$, then $C$ is a twisted algebra of $A$.

Proof. 1. Let $\tau$ be the identity twisting system $\left\{\tau_{g}:=\mathrm{id}_{A} \mid g \in \mathbb{G}\right\}$ where $\mathrm{id}_{A}$ is the identity map of $A$. It is obvious that $\tau$ is a twisting system and $A=A^{\tau}$.

2. Let $B$ be a twisted algebra $A^{\tau}$ for some $\tau=\left\{\tau_{g} \mid g \in \mathbb{G}\right\}$. Define $\tau^{-1}=$ $\left\{\tau_{g}^{-1} \mid g \in \mathbb{G}\right\}$. It is called the inverse twisting system of $\tau$. Since $\tau_{g}$ is a graded $k$-linear map, so is $\tau_{g}^{-1}$. We need to prove that $\tau^{-1}$ is a twisting system of $B$ and that $A=B^{\tau^{-1}}$. Let $*$ denote the multiplication of $B=A^{\tau}$. For every $y \in A_{h}\left(=B_{h}\right)$, $z \in A_{l}\left(=B_{l}\right)$, by $(2.1 .3)$,

$$
\tau_{g}^{-1}(y) * \tau_{g h}^{-1}(z)=\tau_{g}^{-1}(y) \tau_{h} \tau_{g h}^{-1}(z)=\tau_{g}^{-1}(y z)=\tau_{g}^{-1}\left(y * \tau_{h}^{-1}(z)\right)
$$

Hence $\tau^{-1}$ is a twisting system of $B$ and $B^{\tau^{-1}}$ is well-defined. The algeioias $A$ and $B^{\tau^{-1}}$ have the same underlying $k$-module $\bigoplus_{g} A_{g}$. It remains to prove that they have the same multiplication. Let $\bullet$ be the multiplication of $B^{\tau^{-1}}$. For all $y \in A_{h}$, $z \in A_{l}$,

$$
y \cdot z=y * \tau_{h}^{-1}(z)=y \tau_{h} \tau_{h}^{-1}(z)=y z .
$$

Hence $A$ and $B^{\tau^{-1}}$ have the same multiplication. Thus $A=B^{\tau^{-1}}$. 
3. Let $B$ be a twisted algebra $A^{\tau}$ for some $\tau=\left\{\tau_{g} \mid g \in \mathbb{G}\right\}$, and let $C$ be a twisted algebra $B^{\tau^{\prime}}$ for some $\tau^{\prime}=\left\{\tau_{g}^{\prime} \mid g \in \mathbb{G}\right\}$. Let $*$ and - denote the multiplications of $B$ and $C$ respectively. Algebras $A, B$ and $C$ have the same underlying $k$-module $\bigoplus_{g} A_{g}$. Define $\tau_{g}^{\prime \prime}=\tau_{g} \tau_{g}^{\prime}$ and $\tau^{\prime \prime}=\left\{\tau_{g}^{\prime \prime} \mid g \in \mathbb{G}\right\}$, which is called the convolution product of $\tau$ and $\tau^{\prime}$. We need to prove that $\tau^{\prime \prime}$ is a twisting system of $A$ and $A^{\tau^{\prime \prime}}=C$. For all $y \in A_{h}, z \in A_{l}$,

$$
\begin{aligned}
\tau_{g} \tau_{g}^{\prime}\left(y \tau_{h} \tau_{h}^{\prime}(z)\right) & =\tau_{g} \tau_{g}^{\prime}\left(y * \tau_{g}^{\prime}(z)\right)=\tau_{g}\left(\tau_{g}^{\prime}(y) * \tau_{g h}^{\prime}(z)\right) \\
& =\tau_{g}\left(\tau_{g}^{\prime}(y) \tau_{h} \tau_{g h}^{\prime}(z)\right)=\tau_{g} \tau_{g}^{\prime}(y) \tau_{g h} \tau_{g h}^{\prime}(z) .
\end{aligned}
$$

Hence $\tau^{\prime \prime}$ is a twisting system of $A$. Let $\circ$ denote the multiplication of $A^{t^{\prime \prime}}$. For every $y \in A_{h}, z \in A_{l}$,

$$
y \circ z=y \tau_{h} \tau_{h}^{\prime}(z)=y * \tau_{h}^{\prime}(z)=y \bullet z .
$$

Hence $A^{t^{\prime \prime}}$ and $C$ have the same multiplication. Therefore $A^{\tau^{\prime \prime}}=C$.

By using twisting systems, we can define twisted modules of graded right modules in a similar way to that of defining twisted algebras of graded algebras. But the definition stated next does not work for graded left modules. We need a left-hand version of a twisting system to define a twisted module of a left module (for details see § 4 ).

Proposition and Definition 2.6. Let $A$ be a graded algebra, and let $\tau=$ $\left\{\tau_{g} \mid g \in \mathbb{G}\right\}$ be a twisting system of $A$ and $A^{\tau}$ be the corresponding twisted algebra of $A$. Suppose that $M=\bigoplus_{g} M_{g}$ is a graded right A-module. Then there is a graded right $A^{\tau}$-module structure, which is also denoted by $*$, on the underlying graded $k$-module $\bigoplus_{g} M_{g}$ defined by

$$
m * z=m \tau_{h}(z)
$$

for all $m \in M_{h}, z \in A_{l}$. The graded right $A^{\tau}$-module $\left(\bigoplus_{g} M_{g}, *\right)$ is called a twisted module of $M$ by $\tau$ and it is denoted by $M^{\tau}$.

Proof. By definition, $*$ is a $k$-bilinear map from $M \otimes_{k} A$ to $M$. For every $m \in M_{g}, y \in A_{h}$ and $z \in A_{l}$,

$$
(m * y) * z=\left(m \tau_{g}(y)\right) \tau_{g h}(z)=m\left(\tau_{g}(y) \tau_{g h}(z)\right)=m \tau_{g}\left(y \tau_{h}(z)\right)=m *(y * z),
$$

where only the last * represents the multiplication of $A^{\tau}$. Hence $M^{\tau}=\left(\oplus_{g} M_{g}, *\right)$ is a graded right $A^{\tau}$-module.

An analogue of Proposition 2.5 holds for graded right modules. The proof is similar to that of Proposition 2.5 and is omitted.

Proposirion 2.7. Let $A$ be a graded algebra, and let $M$ be a graded right $A$-module. Then the following statements are true.

1. (Reflexivity) $M$ is a twisted module of itself.

2. (Symmetry) if $N$ is a twisted module of $M$, then $M$ is a twisted module of $N$. 
3. (Transitivity) if $N$ is a twisted module of $M$ and $L$ is a twisted module of $N$, then $L$ is a twisted module of $M$.

Proposition 2.8. 1. A graded algebra $B$ is isomorphic to a twisted algebra of $A$ if and only if there is a set of graded $k$-linear bijections $\left\{\phi_{g} \mid g \in \mathbb{G}\right\}$ from $B$ to $A$ which satisfy

$$
\phi_{g}(a b)=\phi_{g}(a) \phi_{a h}(b)
$$

for all $a \in B_{h}, b \in B_{l}$.

2. The graded algebra $B$ is isomorphic to a twisted algebra of $A$ if and only if $A$ is isomorphic to a twisted algebra of $B$.

Proof. Suppose that $f$ is a graded algebra isomorphism from $B$ to $A^{\tau}$ where $\tau=\left\{\tau_{g} \mid g \in \mathbb{G}\right\}$ is a twisting system of $A$. Then we have

$$
\tau_{g} f(a b)=\tau_{g}(f(a) * f(b))=\tau_{g}\left(f(a) \tau_{h} f(b)\right)=\tau_{g} f(a) \tau_{g h} f(b) .
$$

Hence the set $\left\{\phi_{g}:=\tau_{g} f \mid g \in \mathbb{G}\right\}$ satisfies (2.8.1).

Conversely, we suppose that $\left\{\phi_{g} \mid g \in \mathbb{G}\right\}$ is a set of graded $k$-linear bijections from $B$ to $A$ satisfying (2.8.1). Define $\tau=\left\{\tau_{g}:=\phi_{g} \phi_{e}^{-1} \mid g \in \mathbb{G}\right\}$. It suffices to prove that $\tau$ is a twisting system of $A$, and that $\phi_{e}$ is a graded algebra isomorphism from $B$ to $A^{\tau}$. Let $y=\phi_{g}(a)$ and $z=\phi_{g h}(b)$. Applying $\phi_{g}^{-1}$ on both sides of (2.8.1), we obtain

$$
\phi_{g}^{-1}(y z)=\phi_{g}^{-1}(y) \phi_{g h}^{-1}(z)
$$

for all $y \in A_{h}, z \in A_{l}$. By (2.8.1), and (2.8.2) at $g=e$, we have

$$
\begin{aligned}
\phi_{g} \phi_{e}^{-1}\left(y \phi_{h} \phi_{e}^{-1}(z)\right) & =\phi_{g}\left(\phi_{e}^{-1}(y) \phi_{e h}^{-1} \phi_{h} \phi_{e}^{-1}(z)\right) \\
& =\phi_{g}\left(\phi_{e}^{-1}(y) \phi_{e}^{-1}(z)\right)=\phi_{g} \phi_{e}^{-1}(y) \phi_{g h} \phi_{e}^{-1}(z)
\end{aligned}
$$

for all $y \in A_{h}, z \in A_{l}$. Hence $\tau$ is a twisting system. Let $*$ denote the multiplication of $A^{\tau}$. By (2.8.1),

$$
\phi_{e}(a b)=\phi_{e}(a) \phi_{h}(b)=\phi_{e}(a) \phi_{h} \phi_{e}^{-1} \phi_{e}(b)=\phi_{e}(a) * \phi_{e}(b)
$$

for all $a \in B_{h}, b \in B_{l}$. Therefore $\phi_{e}$ is a graded algebra isomorphism from $B$ to $A^{\text {t }}$. Part 2 follows from Part 1 and (2.8.2).

To conclude this section we give the following examples.

EXAMPLE 2.9: semigroup algebras and their twisted algebras. Let $\mathbb{G}$ be a semigroup and $R$ be a $k$-algebra. The semigroup algebra $R \mathbb{G}$ is a free $R$-module $\bigoplus_{g} R g$ with multiplication $\left(r_{1} g\right)\left(r_{2} h\right)=\left(r_{1} r_{2}\right)(g h)$ for all $r_{1}, r_{2} \in R$ and $g, h \in \mathbb{G}$. We may identify $R$ with a subring $R e$ and identify $\mathbb{G}$ with a set of elements $\left\{\bar{g}:=1_{R} g \mid g \in \mathbb{G}\right\}$. Then $r \bar{g}=\bar{g} r$ for all $r \in R, g \in \mathbb{G}$ and $\bar{g} \bar{h}=\overline{g h}$ for all $g, h \in \mathbb{G}$. The identity of $R G$ is equal to the identity $1_{R}$ of $R$ and the unit $e$ of $\mathbb{G}$. An algebra $B$ is called a crossed product of $R$ by $G$ if

(a) $R$ is a $k$-subalgebra of $B$ and $\bar{G}=\{\bar{g} \mid g \in \mathbb{G}\}$ is a set of elements of $B$ such that both $1_{R}$ and $\bar{e}$ are equal to the identity of $B$,

(b) $R \bar{g}=\bar{g} R$ for all $g \in \mathbb{G}$ and $R \bar{g} R \bar{h}=R \overline{g h}$ for all $g, h \in \mathbb{G}$, and 
(c) $B=\bigoplus_{g} R \bar{g}=\bigoplus_{g} \bar{g} R$ is a free left and right $R$-module.

As a consequence, $B$ is a $\mathbb{G}$-graded $k$-algebra with $\operatorname{deg}(\bar{g})=g$. Let $\cdot$ denote the multiplication of $B$. In general, $r \cdot \bar{g} \neq \bar{g} \cdot r$ for $r \in R, g \in \mathbb{G}$ and $\bar{g} \cdot \bar{h} \neq \overline{g h}$ for $g, h \in \mathbb{G}$. A special case is when $r \cdot \bar{g}=\bar{g} \cdot r$ for all $r \in R, g \in \mathbb{G}$, and such $B$ is called a twisted semigroup algebra. (This 'twisted' is not the 'twisted' in Definition 2.3 , but we will see that a crossed product of $R$ by $\mathbb{G}$ is a twisted algebra of a semigroup algebra in the sense of Definition 2.3.) If $\mathbb{G}$ is a group, these definitions can be found in $[12, \S 1.5]$. Let $B$ be a crossed product of $R$ by $\mathbb{G}$. For any $h, l \in \mathbb{G}$, we define a $k$-linear map $f_{h, l}$ from $R$ to itself by

$$
\bar{h} \cdot r \bar{l}=f_{h, l}(r) \overline{h l}
$$

for all $r \in R$. It is obvious that $f_{h, l}$ is a bijection and the multiplication of $r_{1} \bar{h}$ and $r_{2} \bar{l}$ in $B$ can be written as

$$
r_{1} \bar{h} \cdot r_{2} \bar{l}=r_{1} f_{h, l}\left(r_{2}\right) \overline{h l} .
$$

For all $g, h, l \in \mathbb{G}$ and $r_{1}, r_{2} \in R$, by associativity, we have

and

$$
\bar{g} \cdot r_{1} \bar{h} \cdot r_{2} \bar{l}=\left(\bar{g} \cdot r_{1} \bar{h}\right) \cdot r_{2} \bar{l}=f_{g, h}\left(r_{1}\right) \overline{g h} \cdot r_{2} \bar{l}=f_{g, h}\left(r_{1}\right) f_{g h, l}\left(r_{2}\right) \overline{g h l},
$$

$$
\bar{g} \cdot r_{1} \bar{h} \cdot r_{2} \bar{l}=\bar{g} \cdot\left(r_{1} \bar{h} \cdot r_{2} \bar{l}\right)=\bar{g} \cdot r_{1} f_{h, l}\left(r_{2}\right) \overline{h l}=f_{g, h l}\left(r_{1} f_{h, l}\left(r_{2}\right)\right) \overline{g h l} .
$$

Since $B$ is a free $R$-module, we obtain that

$$
f_{g . h}\left(r_{1}\right) f_{g h . l}\left(r_{2}\right)=f_{g, h l}\left(r_{1} f_{h, l}\left(r_{2}\right)\right)
$$

for all $g, h, l \in \mathbb{G}$ and $r_{1}, r_{2} \in R$. Now we define a graded $k$-module automorphism $\tau_{g}$ of the semigroup algebra $R \mathbb{G}$ by $\tau_{g}(r h)=f_{g, h}(r) h$ for all $r \in R$ and $h \in \mathbb{G}$. Let $\tau$ be the set $\left\{\tau_{g} \mid g \in \mathbb{G}\right\}$. We claim that $\tau$ is a twisting system of $R \mathbb{G}$ and the twisted algebra $(R \mathbb{G})^{t}$ is the crossed product $B$. For every $g, h, l \in \mathbb{G}$ and $r_{1}, r_{2} \in R$, we have

and

$$
\tau_{g}\left(r_{1} h \tau_{h}\left(r_{2} l\right)\right)=\tau_{g}\left(r_{1} f_{h . l}\left(r_{2}\right) h l\right)=f_{g, h l}\left(r_{1} f_{h, l}\left(r_{2}\right)\right) h l
$$

$$
\tau_{g}\left(r_{1} h\right) \tau_{g h}\left(r_{2} l\right)=f_{g, h}\left(r_{1}\right) h f_{g h, l}\left(r_{2}\right)=f_{g, h}\left(r_{1}\right) f_{g h . l}\left(r_{2}\right) h l .
$$

By (2.9.2), $\tau_{g}\left(r_{1} h \tau_{h}\left(r_{2} l\right)\right)=\tau_{g}\left(r_{1} h\right) \tau_{g h}\left(r_{2} l\right)$. Hence $\tau$ is a twisting system of the semigroup algebra $R \mathbb{G}$. Let $*$ be the multiplication of the twisted algebra $(R \mathbb{G})^{\tau}$. Then

$$
r_{1} h * r_{2} l=r_{1} h \tau_{h}\left(r_{2} l\right)=r_{1} h f_{h, l}\left(r_{2}\right) l=r_{1} f_{h, l}\left(r_{2}\right) h l .
$$

Combining with (2.9.1), we see that the multiplications of the twisted algebra $(R \mathbb{G})^{\tau}$ and the algebra $B$ are the same. Hence $(R \mathbb{G})^{\tau}=B$. Therefore every crossed product of $R$ by $\mathbb{G}$ is a twisted algebra of the semigroup algebra $R \mathbb{G}$. Conversely, let $B$ be a twisted algebra of the semigroup algebra $R \mathbb{G}$ by a twisting system $\tau=\left\{\tau_{g} \mid g \in \mathbb{G}\right\}$. By Proposition 2.4, we may assume that $\tau_{e}$ is the identity map of $R \mathbb{G}$. Then $R \cong R e$ is still a subring of $B$ and $\left\{\bar{g}:=1_{R} g \in B \mid g \in \mathbb{G}\right\}$ is a set of elements of $B$. Let $*$ be the multiplication of $B$. For all $g, h \in \mathbb{G}$,

$$
R * \bar{g}=R \tau_{e}(\bar{g})=R \bar{g}=\bar{g} R=\bar{g} * \tau_{g}^{-1}(R)=\bar{g} * R
$$


and

$$
(R * \bar{g}) *(R * \bar{h})=R \bar{g} * R \bar{h}=R \bar{g} \tau_{g}(R \bar{h})=R \bar{g} R \bar{h}=R \overline{g h}=R * \tau_{e}^{-1}(\overline{g h})=R * \overline{g h} .
$$

Since the algebra $R G=\bigoplus_{g} R g=\bigoplus_{g} g R$ is a free right and left $R$-module, the algebra $B=\bigoplus_{g \in \mathbb{G}} R * \bar{g}=\bigoplus_{g \in \mathbb{G}} \bar{g} * R$ is a free right and left $R$-module. Therefore $B$ is a crossed product of $R$ by $\mathbb{G}$. Thus we have proved the following theorem.

Proposition 2.10. $A \mathbb{G}$-graded algebra $B$ is isomorphic to a crossed product of $R$ by $\mathbb{G}$ if and only if $B$ is isomorphic to a twisted algebra of the semigroup algebra $R G$.

EXAMPLE 2.11: a special case of Example 2.9. Let $\mathbb{Z}_{2}$ denote both the finite abelian group $\mathbb{Z} / 2 \mathbb{Z}$ and the finite field $\mathbb{Z} / 2 \mathbb{Z}$, and let $k=\mathbb{Z}_{2}(t)$ be the field of rational functions of an indeterminate $t$ over the base field $\mathbb{Z}_{2}$. For every $r \in k-\{0\}$, the $\mathbb{Z}_{2}$-graded $k$-algebra $A_{r}:=k[x] /\left(x^{2}+r\right)$ is a crossed product of $k$ by $\mathbb{Z}_{2}$. The group algebra $k \mathbb{Z}_{2}$ is $A_{-1}=k[x] /\left(x^{2}-1\right)$. Therefore $A_{r}$ is a twisted algebra of $A_{-1}$ for every $r \in k-\{0\}$. In the algebra $A_{-1}=k[x] /\left(x^{2}-1\right)$,

$$
(x+1)^{2}=x^{2}+2 x+1=1+1+2 x=2(x+1)=0 .
$$

Hence $A_{-1}$ is not semiprime. It is also easy to check that every graded algebra automorphism of $A_{-1}$ is the identity map of $A_{-1}$. Hence there are no proper algebraic twisting systems of $A_{-1}$. Suppose that $r$ is not a square of an element in the field $k=\mathbb{Z}_{2}(t)$. Then $x^{2}+r$ is an irreducible polynomial of $x$ over $k$ and $A_{r}=k[x] /\left(x^{2}+r\right)$ is a field. Hence $A_{r}$ is not isomorphic to $A_{-1}$. Therefore not every proper twisting system is an algebraic twisting system.

EXAMPLE $2.12[3, \S 8]$ : twisting systems induced by homogeneous regular normal elements of degree 1 . Let $A$ be a $\mathbb{Z}$-graded algebra and let $x$ be a homogeneous regular normal element of degree 1 . Then there is graded algebra automorphism $f$ of $A$ such that $x f(a)=a x$ for all $a \in A$. Since $x x=x x, f(x)=x$. Let $\tau$ be the twisting system $\left\{f^{n} \mid n \in \mathbb{Z}\right\}$. In the twisted algebra $A^{\tau}$, we have

$$
a * x=a f^{n}(x)=a x \text { and } x * a=x f(a)=a x .
$$

Hence $a * x=x * a$ for all homogeneous elements $a$. Then $x$ is central (and regular) in $A^{\tau}$.

For example, let $A$ be the Ore extension $B[x, \sigma]$ where $B$ is a $\mathbb{Z}$-graded algebra and where $\sigma$ is a graded automorphism of $B$. Suppose $\operatorname{deg}(x)=1$. The element $x$ is regular and normal, and the twisted algebra of $B[x, \sigma]^{\tau}$ defined above is isomorphic to $B^{\sigma^{-1}}[x]$ where $B^{\sigma^{-1}}$ is the $\mathbb{Z}$-graded twisted algebra of $B$ by the twisting system $\left\{\sigma^{-n} \mid n \in \mathbb{Z}\right\}$. If $B=B_{0}$, then $B^{\sigma^{-1}}=B$. In this case, $B[x, \sigma]$ is a twisted algebra of the polynomial extension $B[x]$.

\section{Equivalences of graded categories}

In this section we investigate the relationship between twisted algebras and equivalences of categories of graded modules, and prove Theorem 1.1, Theorem 1.2 and Theorem 1.4 stated in the introduction. Let $A$ be a $\mathbb{G}$-graded $k$-algebra. We denote by $\mathrm{Gr}-A$ the category of $\mathbb{G}$-graded right $A$-modules, with morphisms 
being graded homomorphisms (of degree $e$ ). Then $\mathrm{Gr}-A$ is a $k$-linear abelian category. The following is Theorem 1.1 for $\mathbb{G}$-graded algebras.

THEOREM 3.1. Let $A$ and $B$ be two G-graded algebras. If $B$ is isomorphic to a twisted algebra of $A$, then the categories $\mathrm{Gr}-A$ and $\mathrm{Gr}-B$ are isomorphic.

Proof. If $B$ is isomorphic to $A$, then $\mathrm{Gr}-A$ and $\mathrm{Gr}-B$ are obviously isomorphic. So we may assume that $B$ is a twisted algebra $A^{\tau}$ for some $\tau=\left\{\tau_{g} \mid g \in \mathbb{G}\right\}$. Define a functor $F$ from $\mathrm{Gr}-A$ to $\mathrm{Gr}-A^{\tau}$ as follows: for every graded right $A$-module $M$, $F(M)=M^{\tau}$, and for every graded homomorphism $\psi$ from $M$ to $N, F(\psi)=\psi$. To prove that $F$ is well-defined, it suffices to check that $\psi$ is also a graded homomorphism from $M^{\tau}$ to $N^{\tau}$. Since $M$ and $M^{\tau}$ have the same underlying graded $k$-module (and similarly for $N$ and $N^{\tau}$ ), $\psi$ is a graded $k$-linear map from $M^{\tau}$ to $N^{\tau}$. For every $m \in M_{h}$ and $z \in A_{l}$,

$$
\psi(m * z)=\psi\left(m \tau_{h}(z)\right)=\psi(m) \tau_{h}(z)=\psi(m) * z
$$

where $*$ is defined in (2.6.1). Hence $\psi$ is a graded $A^{\tau}$-module homomorphism from $M^{\tau}$ to $N^{\tau}$ if (and only if) it is a graded $A$-module homomorphism from $M$ to $N$. Therefore $F$ is well-defined.

By Proposition 2.5.2, $A$ is a twisted algebra of $A^{\tau}$ by the inverse twisting system $\tau^{-1}$. Thus we can define a functor $G$ from Gr- $A^{\tau}$ to $\mathrm{Gr}-A$ in the same way as we define $F$. By Proposition 2.7.3, for every graded $A$-module $M, G F(M)=$ $\left(M^{\tau}\right)^{\tau^{-1}}=M$, and for every graded $A^{\tau}$-module $M^{\prime}, F G\left(M^{\prime}\right)=\left(M^{\prime \tau^{-1}}\right)^{\tau}=M^{\prime}$. Hence $G F$ is the identity functor on $\mathrm{Gr}-A$ and $F G$ is the identity functor on $\mathrm{Gr}-A^{\tau}$. Therefore, the categories $\mathrm{Gr}-A$ and $\mathrm{Gr}-A^{\tau}$ are isomorphic.

By the construction of $F$ and $G, F(A)=A^{\tau}$ and $G\left(A^{\tau}\right)=A$. Then $F$ is not a graded Morita equivalence functor unless $A$ is isomorphic to $A^{\tau}$. In other words, if $A$ and $B$ are graded Morita equivalent, then $B$ is hardly ever isomorphic to a twisted algebra of $A$.

Our next aim is to investigate when the converse of Theorem 3.1 holds. Let us first recall the usual shift operators on graded modules. Let $M=\bigoplus_{g \in \mathbb{G}} M_{g}$ be a graded right $A$-module, and let $h$ be an element of $\mathbb{G}$. We define a graded right $A$-module $M[h]$ by setting $(M[h])_{g}=M_{h g}$, that is, the homogeneous component of $M[h]$ of degree $g$ is $M_{h g}$. We usually write $M[h]=\bigoplus_{g} M_{h g}$. The module $M[h]$ is called a shift module of $M$ of degree $h$. As an ungraded module, $M[h]$ is an $A$-submodule of $M$. A corresponding shift operator $s_{h}$ from $\mathrm{Gr}-A$ to itself is defined as follows: for every graded right $A$-module $M, s_{h}(M)=M[h]$, and for every graded $A$-module homomorphism $\psi, s_{h}(\psi)=\left.\psi\right|_{M[h]}$.

The shift functor $s_{e}$, where $e$ is the unit of $\mathbb{G}$, is the identity functor on Gr- $A$. For all $g, h \in \mathbb{G}, s_{g} s_{h}=s_{h g}$ (not $s_{g h}$ !). If $G$ is a group, $s_{g}$ has the inverse functor $s_{g}{ }^{-1}$ and then $s_{g}$ is an automorphism of $\mathrm{Gr}-A$. However, if $\mathbb{G}$ is only a semigroup, then the functor $s_{h}$ may not be faithful and full.

Now let $\mathbb{G}$ be a left cancellative semigroup, that is, $g h_{1}=g h_{2}$ implies $h_{1}=h_{2}$ for all $g, h_{1}, h_{2} \in \mathbb{G}$. Let $M=\bigoplus_{g} M_{g}$ be a graded right $A$-module, and let $h$ be an element of $\mathbb{G}$. We define a graded right $A$-module $M\left[h^{-1}\right]$ by setting $\left(M\left[h^{-1}\right]\right)_{g}=$ $M_{l}$ if $g=h l$ and $\left(M\left[h^{-1}\right]\right)_{g}=0$ otherwise. If $h$ has an inverse in $G$, then $M\left[h^{-1}\right]=\bigoplus_{g} M_{h^{-1}}$, which is the usual shift module of $M$ of degree $h^{-1}$. If $h$ has no inverse in $G$, then $h^{-1}$ is only a formal symbol. The graded right $A$-module 
$M\left[h^{-1}\right]$ is called a shift module of $M$ of degree $h^{-1}$. If we remove the grading, then $M\left[h^{-1}\right]=M$ as a right $A$-module, for every $h$. The corresponding shift operator $s_{h^{-1}}$ from $\mathrm{Gr}-A$ to itself is defined as follows: for every graded right $A$-module $M$, $s_{h^{-1}}(M)=M\left[h^{-1}\right]$, and for every graded $A$-module homomorphism $\psi, s_{h^{-1}}(\psi)=\psi$. It is easy to check the following properties:

(i) $s_{h^{-1}}$ is a faithful, full and exact functor for every $h \in \mathbb{G}$,

(ii) $s_{h^{-1} l_{l^{-1}}}=s_{(h l)^{-1}}$ for all $h, l \in \mathbb{G}$, and

(iii) the image of $s_{h^{-1}}$ is a full subcategory of all graded right $A$-modules $M=\bigoplus_{g} M_{g}$ such that $M_{g}=0$ for all $g \notin h \mathbb{G}$.

Definition 3.2. Let $\mathscr{C}$ be an abelian category and let $\mathscr{S}:=\left\{S_{g} \mid g \in \mathbb{G}\right\}$ be a set of functors from $\mathscr{C}$ to itself. Then $\mathscr{S}$ is called a shift of $\mathscr{C}$ if

(s1) $S_{e}$ is the identity functor on $\mathscr{C}$,

(s2) $S_{g} S_{h}=S_{g h}$ for all $g, h \in \mathbb{G}$,

(s3) $S_{g}$ is a faithful and full functor for every $g \in \mathbb{G}$.

If $\mathscr{S}$ is a shift of $\mathscr{C}$, then the pair $(\mathscr{C}, \mathscr{S})$ (or the category $\mathscr{C}$ alone) is called a G-graded category.

Note that if $\mathbb{G}$ is a group, then (s3) is a consequence of (s1) and (s2). If $\mathbb{G}$ is a left cancellative semigroup, then $\left\{S_{g}:=s_{g^{-1}} \mid g \in \mathbb{G}\right\}$ is a shift of the category Gr- $A$. If $\mathbb{G}$ is an abelian group, then both $\left\{s_{g} \mid g \in \mathbb{G}\right\}$ and $\left\{s_{g}-1 \mid g \in \mathbb{G}\right\}$ are shifts of Gr- $A$. However, if $\mathbb{G}$ is a non-abelian group, then $\left\{s_{g} \mid g \in \mathbb{G}\right\}$ is not a shift of Gr- $A$ in the sense of Definition 3.2 because $s_{g} s_{h}=s_{h g} \neq s_{g h}$ for some $g, h \in \mathbb{G}$. From now on, the shift of Gr- $A$ always means the set $\left\{S_{g}:=s_{g^{-1}} \mid g \in \mathbb{G}\right\}$. Let $\mathscr{C}$ be a $\mathbb{G}$-graded $k$-linear abelian category with the shift $\mathscr{S}=\left\{S_{g} \mid g \in \mathbb{G}\right\}$ and let $M$ be an object of $\mathscr{C}$. We define

$$
\Gamma(M)=\bigoplus_{g \in \mathbb{G}} \operatorname{Hom}_{\mathscr{G}}\left(S_{g}(M), M\right) .
$$

Since $\mathscr{C}$ is $k$-linear, $\operatorname{Hom}_{\mathscr{C}}\left(S_{g}(M), M\right)$ has a natural $k$-module structure for each $g$, and $\operatorname{Hom}_{\mathscr{E}}\left(S_{e}(M), M\right)=\operatorname{Hom}_{\mathscr{G}}(M, M)$ is a $k$-algebra. Therefore $\Gamma(M)$ is a $\mathbb{G}$-graded $k$-module. For all $a \in \operatorname{Hom}_{\mathscr{E}}\left(S_{g}(M), M\right)$ and $b \in \operatorname{Hom}_{\mathscr{E}}\left(S_{h}(M), M\right)$, we define the multiplication to be the composition of morphisms of $a$ and $S_{g}(b)$, that is, $a b=a S_{g}(b)$. Since $S_{g} S_{h}=S_{g h}$, the multiplication of $\Gamma(M)$ is associative. Thus $\Gamma(M)$ becomes a $\mathbb{G}$-graded $k$-algebra. Given two $\mathbb{G}$-graded categories $\mathscr{C}$ and $\mathscr{D}$, we may use the same $\mathscr{S}=\left\{S_{g} \mid g \in \mathbb{G}\right\}$ for the shift of both $\mathscr{C}$ and $\mathscr{D}$.

THEOREM 3.3. Let $\mathscr{C}$ and $\mathscr{D}$ be two $\mathbb{G}$-graded $k$-linear abelian categories with a shift $\mathscr{S}=\left\{S_{g} \mid g \in \mathbb{G}\right\}$, and let $M$ be an nbiect of $\mathscr{C}$ and $N$ an object of $\mathscr{D}$. If there is a faithful and full functor $F$ from $\mathscr{C}$ to $\mathscr{D}$ such that $F\left(S_{g}(M)\right) \cong S_{g}(N)$ for all $g \in \mathbb{G}$, then $\Gamma(M)$ is isomorphic to a twisted algebra of $\Gamma(N)$ as $\mathbb{G}$-graded $k$-algebras.

Proof. By Proposition 2.8, it suffices to construct a set of graded $k$-linear isomorphisms $\left\{\phi_{g}\right\}$ from $\Gamma(N)$ to $\Gamma(M)$ which satisfies (2.8.1).

Let $t_{g}$ denote the isomorphism from $F\left(S_{g}(M)\right)$ to $S_{g}(N)$ for every $g \in \mathbb{G}$. For 
every $g \in \mathbb{G}$, we define a map $\phi_{g}$ from $\Gamma(N)$ to $\Gamma(M)$ as follows: for each $a \in \operatorname{Hom}_{\mathscr{D}}\left(S_{h}(N), N\right) \subset \Gamma(N)$,

$$
\phi_{g}(a)=\left(S_{g}\right)^{-1}\left(F^{-1}\left(t_{g}^{-1} S_{g}(a) t_{g h}\right)\right) .
$$

When the map $\phi_{g}$ is applied to $\operatorname{Hom}_{\mathscr{D}}\left(S_{h}(N), N\right)$, it is a composition of the following four $k$-linear maps:

$$
\begin{aligned}
& \text { (m1) } S_{g}: \operatorname{Hom}_{\mathscr{D}}\left(S_{h}(N), N\right) \rightarrow \operatorname{Hom}_{\mathscr{D}}\left(S_{g h}(N), S_{g}(N)\right), \\
& \text { (m2) } t_{g}^{-1}() t_{g h}: \operatorname{Hom}_{\mathscr{D}}\left(S_{g h}(N), S_{g}(N)\right) \rightarrow \operatorname{Hom}_{\mathscr{P}}\left(F\left(S_{g h}(M)\right), F\left(S_{g}(M)\right)\right) \text {, } \\
& \text { (m3) } F^{-1}: \operatorname{Hom}_{\mathscr{D}}\left(F\left(S_{g h}(M)\right), F\left(S_{g}(M)\right)\right) \rightarrow \operatorname{Hom}_{\mathscr{C}}\left(S_{g h}(M), S_{g}(M)\right), \\
& \text { (m4) } S_{g}^{-1}: \operatorname{Hom}_{\mathscr{C}}\left(S_{g h}(M), S_{g}(M)\right) \rightarrow \operatorname{Hom}_{\mathscr{C}}\left(S_{h}(M), M\right) .
\end{aligned}
$$

Since the functors $S_{g}$ and $F$ are faithful and full, the maps (m1), (m3) and (m4) are bijective. The map (m2) is also bijective because $t_{g}$ and $t_{g h}$ are isomorphisms. Hence $\phi_{g}$ is a graded $k$-linear bijection from $\Gamma(N)$ to $\Gamma(M)$. For every $a \in \operatorname{Hom}_{\mathscr{D}}\left(S_{h}(N), N\right), b \in \operatorname{Hom}_{\mathscr{P}}\left(S_{l}(N), N\right)$, we have

$$
\begin{aligned}
\phi_{g}(a b) & =\left(S_{g}\right)^{-1}\left(F^{-1}\left(t_{g}^{-1} S_{g}(a b) t_{g h l}\right)\right) \\
& =\left(S_{g}\right)^{-1}\left(F^{-1}\left(t_{g}^{-1} S_{g}\left(a S_{h}(b)\right) t_{g h l}\right)\right) \\
& =\left(S_{g}\right)^{-1}\left(F^{-1}\left(t_{g}^{-1} S_{g}(a) S_{g h}(b) t_{g h l}\right)\right) \\
& =\left[\left(S_{g}\right)^{-1}\left(F^{-1}\left(t_{g}^{-1} S_{g}(a) t_{g h}\right)\right)\right]\left[\left(S_{g}\right)^{-1}\left(F^{-1}\left(t_{g h}^{-1} S_{g h}(b) t_{g h l}\right)\right)\right] \\
& =\phi_{g}(a) S_{g}^{-1} S_{g h}\left(\phi_{g h}(b)\right) \\
& =\phi_{g}(a) S_{h}\left(\phi_{g h}(b)\right) \\
& =\phi_{g}(a) \phi_{g h}(b) .
\end{aligned}
$$

Hence the set $\left\{\phi_{g} \mid g \in \mathbb{G}\right\}$ satisfies (2.8.1). Therefore $\Gamma(M)$ is isomorphic to a twisted algebra of $\Gamma(N)$.

We return to the category Gr- $A$. The next corollary provides a converse of Theorem 3.1 when $\mathbb{G}$ is left cancellative. However we do not know the answer if $\mathbb{G}$ is not left cancellative.

THEOREM 3.4. Let $\mathbb{G}$ be a left cancellative semigroup and let $A$ and $B$ be two $\mathbb{G}$-graded $k$-algebras. Then $B$ is isomorphic to a twisted algebra of $A$ if and only if there is an equivalence functor $F$ from $\mathrm{Gr}-A$ to $\mathrm{Gr}-B$ such that $F\left(A\left[g^{-1}\right]\right) \cong B\left[g^{-1}\right]$ for all $g \in \mathbb{G}$.

Proof. If $B$ is isomorphic to a twisted algebra $A^{\tau}$, then Gr- $B$ and $\mathrm{Gr}-A^{\tau}$ are isomorphic. Without loss of generality, we may assume that $B$ is the twisted algebra $A^{\tau}$ for some $\tau=\left\{\tau_{g} \mid g=\mathbb{G}\right\}$. By Theorem 3.1, the categories Gr-A and $\mathrm{Gr}-A^{\tau}$ are equivalent. Let $F$ be the equivalence functor from $\mathrm{Gr}-A$ to $\mathrm{Gr}-A^{\tau}$ defined in the proof of Theorem 3.1. It remains to prove that $F\left(A\left[i^{-1}\right]\right)$ $\left(=A\left[i^{-1}\right]^{\tau}\right)$ is isomorphic to $A^{\tau}\left[i^{-1}\right]$ for all $i \in \mathbb{G}$. The right $A^{\tau}$-modules $A\left[i^{-1}\right]^{\tau}$ and $A^{\tau}\left[i^{-1}\right]$ have the same underlying graded $k$-modules $\bigoplus_{g} A\left[i^{-1}\right]_{g}$ where $A\left[i^{-1}\right]_{g}=A_{l}$ if $g=i l$ and $A\left[i^{-1}\right]_{g}=0$ otherwise. Let $\bullet$ and $\circ$ denote the $A^{\tau}$-module structures of $A^{\tau}\left[i^{-1}\right]$ and $A\left[i^{-1}\right]^{\tau}$ respectively. Since $\tau_{i}$ is a graded $k$-linear 
automorphism of $A$, we may regard $\tau_{i}$ as a graded $k$-linear isomorphism from $A^{\tau}\left[i^{-1}\right]$ to $A\left[i^{-1}\right]^{\tau}$. For all $y \in A_{h}=A^{\tau}\left[i^{-1}\right]_{\text {in }}$ and $z \in A_{l}$, we have

$$
\tau_{i}(y \cdot z)=\tau_{i}\left(y \tau_{h}(z)\right)=\tau_{i}(y) \tau_{i h}(z)=\tau_{i}(y) \circ z .
$$

Hence $\tau_{i}$ is a graded $A^{\tau}$-module isomorphism from $A^{\tau}\left[i^{-1}\right]$ to $A\left[i^{-1}\right]^{\tau}$ for every $i \in \mathbb{G}$. Thus $F\left(A\left[i^{-1}\right]\right) \cong A^{\tau}\left[i^{-1}\right]$.

Conversely, suppose that there is an equivalence functor $F$ from $\mathrm{Gr}-A$ to $\mathrm{Gr}-B$ such that $F\left(A\left[g^{-1}\right]\right) \cong B\left[g^{-1}\right]$ for all $g \in \mathbb{G}$. By Theorem 3.3, $\Gamma(B)$ is isomorphic to a twisted algebra of $\Gamma(A)$. It remains to check that $\Gamma(A) \cong A$. For every $a \in \operatorname{Hom}_{\mathrm{Gr} \cdot A}\left(A\left[h^{-1}\right], A\right) \subset \Gamma(A), a$ is a map from $A\left[h^{-1}\right]$ to $A$ and then $a(1) \in A_{h}$. We define a map $f$ from $\Gamma(A)$ to $A$ by $f(a)=a(1)$, and then

$$
f(a b)=f\left(a s_{h^{-1}}(b)\right)=a s_{h^{-1}}(b)(1)=a(b(1))=a(1) b(1)=f(a) f(b) .
$$

Therefore $f$ is a graded algebra isomorphism from $\Gamma(A)$ to $A$. Similarly, $\Gamma(B) \cong B$. Thus $B$ is isomorphic to a twisted algebra of $A$.

By Theorem 3.4, if $F\left(A\left[g^{-1}\right]\right) \cong B\left[g^{-1}\right]$ is automatically true for some class of graded algebras, then $A$ is isomorphic to a twisted algebra of $B$ if and only if $\mathrm{Gr}-A$ is equivalent to $\mathrm{Gr}-B$. This happens if the algebras are connected graded over a field $k$. If $A$ is connected graded, then we can regard $A$ as both a $\mathbb{Z}$-graded algebra and an $\mathbb{N}$-graded algebra. If $B$ is a twisted algebra of $A$ as a $\mathbb{Z}$-graded algebra, then $B$ is a twisted algebra of $A$ as an $\mathbb{N}$-graded algebra. We are now ready to prove Theorem 1.2 from the Introduction.

THEOREM 3.5. Let $A$ and $B$ be two connected graded algebras over a field $k$ with $A_{1} \neq 0$. Then $B$ is isomorphic to a twisted algebra of $A$ if and only if $\mathrm{Gr}-A$ is equivalent to $\mathrm{Gr}-B$.

Proof. One direction is Theorem 3.1. It remains to prove the other direction. There are two cases.

Case 1: $\mathbb{G}=\mathbb{Z}$. In this case objects of the category $\mathrm{Gr}-A$ are $\mathbb{Z}$-graded right $A$-modules. Let $F$ be an equivalence functor from $\mathrm{Gr}-A$ to $\mathrm{Gr}-B$. Since $A$ is connected graded, the only indecomposable graded projective right $A$-modules are $A[n]$ for all $n \in \mathbb{Z}$. The same is true for $B$. Since $F$ is an equivalence, $F(A[n])$ is an indecomposable graded projective right $B$-module. Hence there is an integer, say $f(n)$, such that $F(A[n]) \cong B[f(n)]$. The map $f: n \mapsto f(n)$ is well defined and it certainly is a bijection from $\mathbb{Z}$ to itself. The shift functor $s_{m}$ is an autoequivalence of $\mathrm{Gr}-A$ (and $\mathrm{Gr}-B$ ). Hence we can replace $F$ by $s_{-f(\Omega)} F$ and assume that $F(A) \cong B$. In other words, we may assume that $f(0)=0$. Now we want to prove that $f$ is an identity map of $\mathbb{Z}$. For every $n \in \mathbb{Z}$,

$$
\operatorname{Hom}_{\mathrm{Gr} \cdot A}(A[n], A[n+1]) \cong \operatorname{Hom}_{\mathrm{Gr}-A}(A[-1], A) \cong A_{1} \neq 0 .
$$

Applying $F$ to $A[n]$ and $A[n+1]$, we have

$$
\begin{aligned}
0 \neq \operatorname{Hom}_{\mathrm{Gr} \cdot B}(B[f(n)], B[f(n+1)]) & \cong \operatorname{Hom}_{\mathrm{Gr}-B}(B[f(n)-f(n+1)], B) \\
& \cong B_{f(n+1)-f(n) .}
\end{aligned}
$$

As a consequence, $f(n+1)-f(n) \geqslant 0$. Hence $f(n+1)>f(n)$ because $f$ is a bijection. We have proved that 
(i) $\{f(n) \mid n \in \mathbb{Z}\}=\mathbb{Z}$,

(ii) $f(0)=0$, and

(iii) $\{f(n)\}$ is an increasing sequence of integers.

Therefore $f(n)=n$ and $F(A[n]) \cong B[n]$ for all $n \in \mathbb{Z}$. By Theorem $3.4, B$ is isomorphic to a twisted algebra of $A$.

Case 2: $\mathbb{G}=\mathbb{N}$. In this case objects of the category $\mathrm{Gr}-A$ are $\mathbb{N}$-graded right $A$-modules. It is easy to check that all indecomposable graded projective right $A$-modules are $A[-n]$ for all $n \in \mathbb{N}$. Let $F$ be an equivalence functor from $\mathrm{Gr}-A$ to $\mathrm{Gr}-B$. Then for every $n \in \mathbb{N}, F(A[-n])=B[-f(n)]$ for some $f(n) \in \mathbb{N}$. The argument in Case 1 also works here. Hence we have

(i) $\{f(n) \mid n \in \mathbb{N}\}=\mathbb{N}$, and

(ii) $\{f(n)\}$ is an increasing sequence of integers.

Thus $f(n)=n$ and $F(A[-n]) \cong B[-n]$ for all $n \in \mathbb{N}$. By Theorem $3.4, B$ is isomorphic to a twisted algebra of $A$.

Let us have an example.

EXAMPLE 3.6: twisted algebras of the polynomial ring $k\left[x_{1}, x_{2}\right]$. A natural $\mathbb{Z}$-grading (or $\mathbb{N}$-grading) of $k\left[x_{1}, x_{2}\right]$ is given by $\operatorname{deg}\left(x_{1}\right)=\operatorname{deg}\left(x_{2}\right)=1$. The algebra $k\left[x_{1}, x_{2}\right]$ has two generators $x_{1}$ and $x_{2}$ and one relation $x_{1} x_{2}-x_{2} x_{1}=0$. Then we have the minimal graded projective resolution of the trivial $k\left[x_{1}, x_{2}\right]$ module $k$,

$$
0 \rightarrow k\left[x_{1}, x_{2}\right][-2] \rightarrow k\left[x_{1}, x_{2}\right][-1] \oplus k\left[x_{1}, x_{2}\right][-1] \rightarrow k\left[x_{1}, x_{2}\right] \rightarrow k \rightarrow 0 .
$$

For a connected graded algebra $A, k$ always denotes the trivial module $A / A \geqslant 1$ where $A_{\geqslant 1}=\bigoplus_{n \geqslant 1} A_{n}$. Let $B$ be a twisted algebra of $k\left[x_{1}, x_{2}\right]$ and let $F$ be the equivalence functor from $\operatorname{Gr}-k\left[x_{1}, x_{2}\right]$ to $\mathrm{Gr}-B$. Applying $F$ to the above projective resolution, we obtain a minimal graded projective resolution of the trivial module of $B$,

$$
0 \rightarrow B[-2] \rightarrow B[-1] \oplus B[-1] \rightarrow B \rightarrow k \rightarrow 0 .
$$

Therefore $B$ has two generators, which are $x_{1}$ and $x_{2}$, and one relation. Now what is the relation in the algebra $B$ ? Let $\tau=\left\{\tau_{g} \mid g \in \mathbb{G}\right\}$ be the twisting system of $k\left[x_{1}, x_{2}\right]$ such that $B=k\left[x_{1}, x_{2}\right]^{\tau}$. Then, in the algebra $B$,

$$
x_{1} * \tau_{1}^{-1}\left(x_{2}\right)-x_{2} * \tau_{1}^{-1}\left(x_{1}\right)=x_{1} x_{2}-x_{2} x_{1}=0 \text {. }
$$

Since $\tau_{1}$ is a graded $k$-linear automorphism of $k\left[x_{1}, x_{2}\right]$, there are elements $a, b, c, d \in k$ with $a d-b c \neq 0$ such that

$$
\tau_{1}^{-1}\left(x_{2}\right)=a x_{1}+b x_{2} \text { and } \tau_{1}^{-1}\left(x_{1}\right)=c x_{1}+d x_{2} .
$$

Hence the nnlv relation in $B$ is $a x_{1}^{2}+b x_{1} x_{2}-c x_{2} x_{1}-d x_{2}^{2}=0$. Let $r_{a, b, c, d}$ denote the element $a x_{1}^{2}+b x_{1} x_{2}-c x_{2} x_{1}-d x_{2}^{2}$. Therefore $B$ is isomorphic to the algebra $k\left\langle x_{1}, x_{2}\right\rangle /\left(r_{a, b, c, d}\right)$ where $k\left\langle x_{1}, x_{2}\right\rangle$ is the free algebra generated by $x_{1}$ and $x_{2}$ and where $\left(r_{a, b, c, d}\right)$ is the ideal generated by $r_{a, b, c, d}$. Conversely, let $a, b, c, d$ be four elements in $k$ with $a d-b c \neq 0$. There is a unique graded algebra automorphism $f$ of $k\left[x_{1}, x_{2}\right]$ such that

$$
f_{1}^{-1}\left(x_{2}\right)=a x_{1}+b x_{2} \text { and } f_{1}^{-1}\left(x_{1}\right)=c x_{1}+d x_{2} .
$$


Then $\tau^{\prime}=\left\{f^{n} \mid n \in \mathbb{Z}\right\}$ is an algebraic twisting system of $k\left[x_{1}, x_{2}\right]$ and the twisted algebra $k\left[x_{1}, x_{2}\right]^{\tau^{\prime}}$ is isomorphic to the algebra $k\left\langle x_{1}, x_{2}\right\rangle /\left(r_{a, b . c . d}\right)$. Thus we have proved that an algebra is isomorphic to a twisted algebra of $k\left[x_{1}, x_{2}\right]$ if and only if it is isomorphic to $k\left\langle x_{1}, x_{2}\right\rangle /\left(r_{a, b, c, d}\right)$ for some $a, b, c, d \in k$ with $a d-b c \neq 0$. If we choose $a=d=0, b=p$ and $c=1$, we obtain the skew polynomial ring $k_{p}\left[x_{1}, x_{2}\right]:=k\left\langle x_{1}, x_{2}\right\rangle /\left(p x_{1} x_{2}-x_{2} x_{1}\right)$. If we choose $a=b=c=1$ and $d=0$, we obtain the Jordan polynomial ring $k_{J}\left[x_{1}, x_{2}\right]:=k\left\langle x_{1}, x_{2}\right\rangle /\left(x_{1}^{2}+x_{1} x_{2}-x_{2} x_{1}\right)$. Hence the categories $\mathrm{Gr}-k\left[x_{1}, x_{2}\right], \mathrm{Gr}-k_{p}\left[x_{1}, x_{2}\right]$, and $\mathrm{Gr}-k_{J}\left[x_{1}, x_{2}\right]$ are all equivalent. A similar discussion for the polynomial ring $k\left[x_{1}, \ldots, x_{m}\right]$ is given in $\S 5$.

If the $\mathbb{N}$-grading of $k\left[x_{1}, x_{2}\right]$ is given by $\operatorname{deg}\left(x_{1}\right)=l_{1}>0$ and $\operatorname{deg}\left(x_{2}\right)=l_{2}>0$, we may also work out all twisted algebras of $k\left[x_{1}, x_{2}\right]$. Without loss of generality, we assume that $l_{1} \leqslant l_{2}$. We list the following solutions without proof:

(i) if $l_{1}=l_{2}$, then the solution is similar to that when $l_{1}=l_{2}=1$,

(ii) if $l_{1}<l_{2}$ and $l_{2} / l_{1}$ is not an integer, then every twisted algebra of $k\left[x_{1}, x_{2}\right]$ is isomorphic to $k_{p}\left[x_{1}, x_{2}\right]$ for some non-zero element $p \in k$, and

(iii) if $l_{1}<l_{2}$ and $s:=l_{2} / l_{1}$ is an integer, then every twisted algebra of $k\left[x_{1}, x_{2}\right]$ is isomorphic to $k\left\langle x_{1}, x_{2}\right\rangle /\left(a x_{1}^{s+1}+p x_{1} x_{2}-x_{2} x_{1}\right)$ for some $a, p \in k$ and $p \neq 0$.

Next we are going to prove Theorem 1.4 in the introduction. Let us review some notation. Suppose $A$ is an $\mathbb{N}$-graded noetherian $k$-algebra. A graded $k$-module $M=\bigoplus M_{n}$ is called bounded if $M_{n}=0$ for all $n \gg 0$ and $n \ll 0$, and a graded right $A$-module $M$ is called torsion if every finitely generated submodule of $M$ is bounded. Let Tors denote the full subcategory of $\mathrm{Gr}-A$ consisting of torsion $A$-modules. The quotient category $\mathrm{Gr}-A /$ Tors is denoted by $\mathrm{QGr}-A$. For every module $M$ in $\mathrm{Gr}-A$, the image of $M$ in QGr- $A$ is denoted by $M$. For example, $\mathscr{A}$ is the image of $A_{A}$ in QGr- $A$. The projective scheme of $A$ is defined to be the pair (QGr- $A, \mathscr{A}$ ) which is denoted by $\operatorname{Proj}-A$ (see [4]). It is easy to see that the shift operator $s_{1}$ of Gr- $A$ maps Tors to Tors. Hence $s_{1}$ induces an automorphism of QGr- $A$, which is called a shift operator of QGr- $A$ and is denoted by $s_{1}$ also. We use the notation $M[d]=s_{1}^{d}(M)$ and $\mathcal{M}[d]=s_{1}^{d}(\mathcal{M})$ to denote the $d$ th power of the shift operator. For every object $\mathcal{M}$, it is easy to check that

$$
\bigoplus_{n \in \mathbb{Z}} \operatorname{Hom}_{\mathrm{QGr} \cdot A}(\mathcal{M}, \mathcal{M}[n]) \cong \bigoplus_{n \in \mathbb{Z}} \operatorname{Hom}_{\mathrm{QG} r \cdot A}(\mathcal{M}[-n], \mathcal{M})
$$

as a graded algebra via the map $a \mapsto s_{1}^{-n}(a)$ for all $a \in \operatorname{Hom}_{\mathrm{OG} r \cdot A}(\mathcal{M}, \mathcal{M}[n])$. Note that the right-hand side of (3.6.1) is $\Gamma(\mathcal{M})$ defined in this paper and the left-hand side is $\Gamma(\mathscr{A})$ defined in [4] when $\mathscr{M}=\mathscr{A}$.

THEOREM 3.7. Let $A$ and $B$ be $\mathbb{N}$-graded right noetherian algebras.

1. If $A$ is isomorphic to a twisted algebra of $B$, then there is an equivalence functor $\mathscr{F}$ from $\mathrm{QGr}-A$ to $\mathrm{QGr}-B$ such that $\mathscr{F}(\mathscr{A}[n]) \cong \mathscr{B}[n]$ for all $n \in \mathbb{Z}$.

2. If there is an equivalence functor $\mathscr{F}$ from $\mathrm{QGr}-A$ to $\mathrm{QGr}-B$ such that $\mathscr{F}(\mathscr{A}[n]) \cong \mathscr{B}[n]$ for all $n \in \mathbb{Z}$, then $\Gamma(\mathscr{A})$ is isomorphic to a twisted algebra of $\Gamma(\mathscr{B})$. 
Proof. Part 2 follows from Theorem 3.3 and we only need to prove Part 1 . If $A$ is isomorphic to a twisted algebra of $B$, then by Theorem 3.4 , there is an equivalence functor $F$ from Gr- $A$ to $\mathrm{Gr}-B$ such that $F(A[n])$ is isomorphic to $B[n]$ for all $n \in \mathbb{Z}$. The functor $F$ maps bounded $A$-modules to bounded $B$-modules, so it maps torsion $A$-modules to torsion $B$-modules. Thus we have an induced equivalence functor from QGr- $A$ to $\mathrm{QGr}-B$, which is denoted by $\mathscr{F}$. It follows that the image of $F(M)$ in $\mathrm{QGr}-B$ is isomorphic to $\mathscr{F}(\mathcal{M})$. Therefore we obtain $\mathscr{F}(\mathscr{A}[n]) \cong \mathscr{B}[n]$ for all $n \in \mathbb{Z}$.

To conclude this section we give some obvious equivalences of categories of graded modules in which one algebra is not isomorphic to a twisted algebra of another.

EXAMPLE 3.8: equivalences defined by automorphisms of semigroups. Let $\mathbb{G}$ be a semigroup and let $\sigma$ be an automorphism of $\mathbb{G}$. Let $A=\bigoplus_{g} A_{g}$ be a $\mathbb{G}$-graded algebra. We define $A_{\sigma}$ to be the algebra $\bigoplus_{g} A_{\sigma(g)}$. As an ungraded algebra, $A_{\sigma}=A$, but as a graded algebra $A_{\sigma}$ is not equal to $A$ unless $\sigma$ is the identity map of $\mathbb{G}$. Given a graded right $A$-module, we define $M_{\sigma}$ to be $\bigoplus_{g} M_{\sigma(g)}$. Then $M_{\sigma}$ is a graded right $A_{\sigma}$-module. It is easy to see that $\mathrm{Gr}-A$ is equivalent to $\mathrm{Gr}-A_{\sigma}$ by sending $M$ to $M_{\sigma}$. In general, $A_{\sigma}$ is not isomorphic to a twisted algebra of $A$.

EXAMPLE 3.9: equivalences involving strongly graded algebras. Let $\mathbb{G}$ be a group. A $G$-graded algebra $A=\bigoplus_{g} A_{g}$ is called strongly graded if $A_{g} A_{h}=A_{g h}$ for all $g, h \in \mathbb{G}$. Every crossed product of $R$ by $\mathbb{G}$ is a strongly graded algebra, but not every strongly graded algebra is a crossed product. By [13, Theorem I.3.4], the category $\mathrm{Gr}-A$ is equivalent to $\operatorname{Mod}-A_{e}$ where $\operatorname{Mod}-A_{e}$ is the category of all right $A_{e}$-modules. As a consequence, the category $\mathrm{Gr}-A$ is equivalent to the category $\mathrm{Gr}-A_{e} \mathbb{G}$ where $A_{e} \mathbb{G}$ is the group algebra. By Proposition 2.10, if $A$ is not a crossed product of $A_{e}$ by $\mathbb{G}$, then $A$ is not isomorphic to a twisted algebra of the group algebra $A_{e} \mathbb{G}$.

EXAMPLE 3.10: graded Morita equivalences. Let $A$ be a $\mathbb{Z}$-graded algebra and let $P$ be a graded right progenerator of $A$. Define $B$ to be the graded algebra $\bigoplus_{g} \operatorname{Hom}_{\mathrm{Gr}-A}(P, P[g])$. Then $\mathrm{Gr}-B$ is equivalent to $\mathrm{Gr}-A$. In general, $B$ is not isomorphic to a twisted algebra of $A$.

\section{Other twistings}

Let $A-\mathrm{Gr}$ denote the category of all graded left $A$-modules. In this section we *i!!! prove that if $\tau$ is a twisting system and $\mathbb{G}$ is a group then we can twist graded left moduies as well. As a consequence, we show that if $A^{t}$ is a twisted algebra of $A$ and $\mathbb{G}$ is a group then $A-\mathrm{Gr}$ is equivalent to $A^{\tau}$-Gr. We also briefly discuss twisting systems of a graded coalgebra and the corresponding twisted coalgebras.

From the definitions of a twisting system and a twisted module, it is not obvious that we can construct a 'twisted module' of a left module by a twisting system. We need left-hand analogues of all those concepts. 
Definition 4.1. Let $A$ be a $\mathbb{G}$-graded $k$-algebra. A set of graded $k$-linear automorphisms of $A$, say $\left\{v_{g} \mid g \in \mathbb{G}\right\}$, is called an l-twisting system of $A$ if

$$
v_{l}\left(v_{h}(x) y\right)=v_{h l}(x) v_{l}(y)
$$

for all $g, h, l \in \mathbb{G}$ and all $x \in A_{g}, y \in A_{h}$.

Proposition And Definition 4.2. Let $A$ be a graded algebra, and let $v=$ $\left\{v_{g} \mid g \in \mathbb{G}\right\}$ be an l-twisting system of $A$. Then there is a new graded and associative multiplication $\circ$ on the underlying graded $k$-module $\bigoplus_{g} A_{g}$ defined by

$$
x \circ y=v_{h}(x) y
$$

for all $x \in A_{g}, y \in A_{h}$. The element ${ }_{v} 1:=v_{e}^{-1}(1)$ is the identity with respect to the new graded multiplication $\circ$. The graded algebra $\left(\bigoplus_{g} A_{g}, \circ, 1\right)$ is called the l-twisted algebra of $A$ by $v$ and it is denoted by ${ }^{v} A$.

Let $A^{\text {op }}$ denote the opposite ring of $A$ and let $G^{\text {op }}$ denote the opposite group of $G$. Then $A^{\text {op }}$ is a $\mathbb{G}^{\text {op }}$-graded algebra. It is easy to see that $B$ is an $l$-twisted algebra of $A$ as a $\mathbb{G}$-graded algebra if and only if $B^{\mathrm{op}}$ is a twisted algebra of $A^{\mathrm{op}}$ as a $\mathbb{G}^{\mathrm{op}}$-graded algebra. Hence every concept and every statement in $\$ \S 2$ and 3 has a left-hand analogue. We omit the details here.

The relationship between twisted algebras and $l$-twisted algebras is as follows.

ThEOREM 4.3. Let $G$ be a group and let $A$ be a $\mathbb{G}$-graded algebra. Then a twisted algebra of $A$ is isomorphic to an l-twisted algebra of $A$ (and vice versa).

Proof. Let $B$ be a twisted algebra $A^{\tau}$ for some $\tau=\left\{\tau_{g} \mid g \in \mathbb{G}\right\}$. We define a set of graded $k$-linear automorphisms, say $v=\left\{v_{g} \mid g \in \mathbb{G}\right\}$, by

$$
v_{g}(y)=\tau_{(h g)^{-1}} \tau_{h^{-1}}^{-1}(y)
$$

for all $y \in A_{h}$. Since $\mathbb{G}$ is a group, $(h g)^{-1}$ and $h^{-1}$ are in $\mathbb{G}$ and so $v_{g}$ is well defined. We claim that $v$ is an $l$-twisting system of $A$, and $B$ is isomorphic to the $l$-twisted algebra ${ }^{v} A$. By (2.1.3) and (2.1.1), we have

$$
\begin{aligned}
v_{l}\left(v_{h}(x) y\right) & =\tau_{(g h l)^{-1}} \tau_{(g h)^{-1}}^{-1}\left(\tau_{(g h)^{-1}} \tau_{g^{-1}}^{-1}(x) y\right)=\tau_{(g h l)^{-1}}\left(\tau_{g^{-1}}^{-1}(x) \tau_{g} \tau_{h^{-1}}^{-1}(y)\right) \\
& =\tau_{(g h l)^{-1}} \tau_{g^{-1}}^{-1}(x) \tau_{(h l)^{-1}} \tau_{h^{-1}}^{-1}(y)=v_{h l}(x) v_{l}(y)
\end{aligned}
$$

for all $x \in A_{g}, y \in A_{h}$. Hence $v$ is an $l$-twisting system of $A$. Let $\circ$ denote the multiplication of ${ }^{v} A$. We define a graded $k$-linear map $f$ from $A^{\tau}$ to ${ }^{v} A$ by $f(y)=\tau_{h^{-1}}(y)$ for all $y \in A_{h}$. Then by (2.1.3),

$$
f(y * z)=\tau_{(h l)^{-1}}\left(y \tau_{h}(z)\right)=\tau_{(h l)^{-1}}(y) \tau_{l^{-1}}(z)=\tau_{(h l)^{-1}} \tau_{h^{-1}}^{-1}(f(y)) f(z)=f(y) \circ f(z)
$$

for all $y \in A_{h}, z \in A_{l}$. Therefore $f$ is a graded algebra isomorphism from $A^{\tau}$ to ${ }^{\prime} A$.

Corollary 4.4. 1. Let $\mathbb{G}$ be a group. If $B$ is isomorphic to a twisted algebra of $A$, then $A-\mathrm{Gr}$ and $B-\mathrm{Gr}$ are isomorphic.

2. Let $\mathbb{G}=\mathbb{Z}$. Suppose that $A$ and $B$ are connected graded and $A_{1} \neq 0$. Then $\mathrm{Gr}-A$ and $\mathrm{Gr}-B$ are equivalent if and only if $A-\mathrm{Gr}$ and $B-\mathrm{Gr}$ are equivalent. 
Proof. Part 1 follows from Theorem 4.3 and a left-hand analogue of Theorem 3.1, and Part 2 follows from Theorem 4.3 and Theorem 3.5.

In the case where $\mathbb{G}$ is not a group, we have not found a proof of the statement of Theorem 4.3 and we do not have any counter examples either. We conjecture that Theorem 4.3 holds without the assumption that $\mathbb{G}$ is a group. The statement of Theorem 4.3 holds in the following two cases.

Case 1. Let $B$ be a twisted algebra of $A$ by a twisting system $\tau=\left\{\tau_{g} \mid g \in \mathbb{G}\right\}$. If for all $g, h \in \mathbb{G}, \tau_{g} \tau_{h} \tau_{g}=\tau_{g h g}$, then $B$ is isomorphic to an $l$-twisted algebra of $A$. In particular, if $\tau$ is an algebraic twisting system of $A$, then $v=\left\{v_{g}:=\tau_{g}^{-1} \mid g \in \mathbb{G}\right\}$ is an algebraic $l$-twisting system of $A$, and $A^{\tau}$ is isomorphic to the $l$-twisted algebra " $A$.

Case 2. Let $A$ be a semigroup algebra $R \mathbb{G}$. By Proposition 2.10, a $\mathbb{G}$-graded algebra $B$ is isomorphic to a twisted algebra of $A$ if and only if $B$ is isomorphic to a crossed product of $R$ by $\mathbb{G}$. Symmetrically, a $\mathbb{G}$-graded algebra $B$ is isomorphic to an $l$-twisted algebra of $A$ if and only if $B$ is isomorphic to a crossed product of $R$ by $G$. As a consequence, every twisted algebra of $A$ is isomorphic to an l-twisted algebra of $A$.

In the rest of this section we introduce the definition of twisted coalgebra and state the coalgebra analogue of Theorem 3.1 without proof. The basic facts about coalgebras can be found in [1] and [15]. The concept of coalgebra is a dual concept of algebra. We define a twisted coalgebra of a graded coalgebra in a way dual to that of defining a twisted algebra. A $k$-coalgebra $C$ with comultiplication $\Delta$ is called $G$-graded if

(a) $C=\bigoplus_{g} C_{g}$ where each $C_{g}$ is a $k$-submodule of $C$, and

(b) $\Delta\left(C_{g}\right) \subseteq \sum_{h l=g} C_{h} \otimes_{k} C_{l}$.

Let $C$ be a $\mathbb{G}$-graded $k$-coalgebra and $\tau=\left\{\tau_{g} \mid g \in \mathbb{G}\right\}$ be a set of graded $k$-linear automorphisms of $C$. The set $\tau$ is called a twisting system of $C$ if, for any $g, h \in \mathbb{G}$ and for any $w \in C_{g}$,

$$
\sum_{i} \tau_{h}\left(y_{i}\right) \otimes_{k} \tau_{h l_{i}}\left(z_{i}\right)=\sum_{j} y_{j}^{h} \otimes_{k} \tau_{l_{i}^{h}}\left(z_{j}^{h}\right)
$$

where $\sum_{i} y_{i} \otimes_{k} z_{i}=\Delta(\omega), \quad \sum_{j} y_{j}^{h} \otimes_{k} z_{j}^{h}=\Delta\left(\tau_{h}(w)\right)$ and $l_{i}=\operatorname{deg}\left(y_{i}\right), \quad l_{j}^{h}=\operatorname{deg}\left(y_{j}^{h}\right)$. Given a twisting system $\tau=\left\{\tau_{g} \mid g \in \mathbb{G}\right\}$ of $C$, a new coassociative comultiplication $\Delta_{*}$ on the underlying graded $k$-module $\bigoplus_{g} C_{g}$ is defined by

$$
\Delta_{*}(w)=\sum_{i} y_{i} \otimes_{k} \tau_{l_{1}}\left(z_{i}\right)
$$

where $\sum_{i} y_{i} \otimes_{k} z_{i}=\Delta(w)$ and $l_{i}=\operatorname{deg}\left(y_{i}\right)$. The new graded coalgebra $\left(\bigoplus_{g \in \mathbb{G}} C_{g}, \Delta_{*}\right.$ ) is called a twisted coalgebra of $C$ by $\tau$ and it is denoted by $C^{\tau}$. Let $\varepsilon$ denote the counit (map) of the graded coalgebra $C=\bigoplus_{g} C_{g}$ satisfying $\varepsilon\left(C_{g}\right)=0$ for all $g \neq e$. Then the counit (map) of the twisted coalgebra $C^{\tau}$ is $\varepsilon \tau_{e}^{-1}$. An analogue of Theorem 3.1 holds. Let $C$ be a graded coalgebra. We denote by $\mathrm{Gr}_{\mathrm{co}}-C$ the category of graded right $C$-comodules, with morphisms being graded homomorphisms of comodules of degree $e$.

THEOREM 4.5. Let $C$ and $D$ be two graded coalgebras. If $C$ is isomorphic to a twisted coalgebra of $D$, then the categories $\mathrm{Gr}_{\mathrm{co}}-C$ and $\mathrm{Gr}_{\mathrm{co}}-D$ are isomorphic. 
Twisted coalgebra is a dual concept of twisted algebra. Recall that a graded module $\bigoplus_{g} M_{g}$ is called locally finite if each $M_{g}$ is finite dimensional over a field $k$. If we consider locally finite algebras and coalgebras, then they are also dual to each other in the following sense. Let $M=\bigoplus_{g} M_{g}$ be a locally finite graded $k$-module. We denote by $M^{*}$ the graded $k$-module $\bigoplus_{g} \operatorname{Hom}_{k}\left(M_{g}, k\right)$. If $A$ is a graded algebra, then $A^{*}$ is a graded coalgebra and it is called the dual coalgebra of $A$. Conversely, if $C=\bigoplus_{g} C_{g}$ is a locally finite graded $k$-coalgebra, then $C^{*}$ is a graded algebra and it is called the dual algebra of $C$. If $\tau=\left\{\tau_{g} \mid g \in \mathbb{G}\right\}$ is a twisting system of the graded algebra $A$, then $\tau^{*}:=\left\{\tau_{g}^{*} \mid g \in \mathbb{G}\right\}$ is a twisting system of the graded coalgebra $A^{*}$, and the twisted coalgebra $\left(A^{*}\right)^{\tau^{*}}$ is isomorphic to the dual coalgebra $\left(A^{\tau}\right)^{*}$.

\section{Twisting invariant properties}

In this section we investigate the properties which carry over from a graded algebra to its twisted algebra, and we prove Theorem 1.3 stated in the introduction. As an example, we study the twisted algebras of commutative polynomial rings in some detail. Many concepts with prefix 'graded' are adopted from [13] and we are not going to recall most of them.

A property $\mathscr{P}$ is called $\mathbb{G}$-twisting invariant if a $\mathbb{G}$-graded algebra $A$ has the property $\mathscr{P}$ if and only if every twisted algebra of $A$ has the property $\mathscr{P}$. In the ungraded case, a property is called Morita invariant if it is preserved under Morita equivalence.

If algebras $A$ and $B$ are Morita equivalent, then by [12, Theorem 3.5.9], the centre of $A$ is isomorphic to the centre of $B$. However, for many (probably all) semigroups $\mathbb{G}$, there are examples in which the centre of a $\mathbb{G}$-graded algebra is not isomorphic to the centre of its twisted algebra. Therefore we do not expect any properties related to the centre of an algebra to be $\mathbb{G}$-twisting invariant for any semigroup $\mathbb{G}$. On the other hand, we expect some ' $G$-graded' properties to be G-twisting invariant because

(i) a twisted algebra $A^{\tau}$ is easily constructed from a graded algebra $A$, and

(ii) the categories Gr- $A$ and $\mathrm{Gr}-A^{\tau}$ are equivalent.

However, we are more interested in those properties without the prefix 'G-graded'. By Example 2.11, a $\mathbb{Z}_{2}$-graded algebra $A_{-1}$ is not semiprime and a twisted algebra $A^{\tau}$ of $A_{-1}$ is a field. As an ungraded algebra, $A_{-1}$ is isomorphic to the algebra $k[x] /\left(x^{2}\right)$ where $k$ is the field $\mathbb{Z}_{2}(t)$. Thus the global dimension of $A_{-1}$ is infinite whereas the global dimension of $A^{\tau}$ is zero. Therefore the following properties are not $\mathbb{Z}_{2}$-twisting invariant:

(a) being a division ring,

(b) being a domain,

(c) being a simple ring,

(d) being prime,

(e) being semiprime, and

(f) having finite global dimension.

Note that (c), (d), (e), and (f) are Morita invariant (see, for example, [12, Theorem 3.5.9 and Proposition 3.5.10]). However under some reasonable 
conditions on $\mathbb{G}$ (and on algebras), some Morita invariant properties are twisting invariant.

A group $\mathbb{G}$ is called polycyclic-by-finite if there is a finite chain of subgroups $\{e\}=\mathbb{G}_{0} \subset \mathbb{G}_{1} \subset \ldots \subset \mathbb{G}_{n}=\mathbb{G}$ such that each $\mathbb{G}_{i}$ is a normal subgroup of $\mathbb{G}_{i+1}$ and each factor $\mathbb{G}_{i+1} / \mathbb{G}_{i}$ is either finite or isomorphic to $\mathbb{Z}$. Obvious examples of polycyclic-by-finite groups are finite groups and the group $\mathbb{Z}$. Given an integer $m>0$, let $\mathbb{Z}^{m}$ denote the additive group of the direct sum of $m$ copies of $\mathbb{Z}$. Then $\mathbb{Z}^{m}$ is also polycyclic-by-finite.

Proposition 5.1. Let $\mathbb{G}$ be a subsemigroup of a polycyclic-by-finite group. Then being right noetherian is $\mathbb{G}$-twisting invariant. If, moreover, $\mathbb{G}$ is a group, then being left noetherian is also $\mathbb{G}$-twisting invariant.

Proof. Let $A$ be a right noetherian $\mathbb{G}$-graded algebra and let $B$ be a twisted algebra of $A$. By Theorem 3.4, there is an equivalence functor $F$ from Gr- $A$ to Gr $-B$ such that $F(A)=B$. Hence, $F$ induces a one-to-one correspondence between graded right ideals of $A$ and graded right ideals of $B$ which preserves the containing relation. Hence $A$ being right noetherian implies $B$ being $\mathbb{G}$-graded right noetherian. Let $\mathbb{G}^{\prime}$ be a polycyclic-by-finite group containing $\mathbb{G}$. We can regard $B$ as a $\mathbb{G}^{\prime}$-graded algebra. Then a $\mathbb{G}$-graded right ideal of $B$ is a $\mathbb{G}^{\prime}$-graded right ideal of $B$ (and vice versa). Hence $B$ is $\mathbb{G}^{\prime}$-graded right noetherian. By [14, Theorem 1.3.7], $B$ is ungraded right noetherian. Therefore being right noetherian is $\mathrm{G}$-twisting invariant.

If $\mathbb{G}$ is a group, then $B$ is also an $l$-twisted algebra of $A$. Hence $A$ being left noetherian implies $B$ being left noetherian. Thus being left noetherian is G-twisting invariant.

A semigroup $\mathbb{G}$ is called ordered if there is an order relation $<$ defined on the set $\mathbb{G}$ satisfying

(a) if $g, h \in \mathbb{G}$, then either $g<h$ or $h<g$ or $g=h$,

(b) if $g<h$ and $h<l$, then $g<l$, and

(c) if $g<h$, then $g l<h l$ and $l g<l h$ for all $l \in \mathbb{G}$.

The group $\mathbb{Z}$ and the semigroup $\mathbb{N}$ are naturally ordered. The group $\mathbb{Z}^{m}$ is (lexicographically) ordered.

Proposition 5.2. Suppose that $\mathbb{G}$ is an ordered semigroup. Then being a domain is a G-twisting invariant property.

Proof. Let $A$ be a domain and let $B$ be a twisted algebra of $A$ by a twisting system $\left\{\tau_{g} \mid g \in \mathbb{G}\right\}$ and let $*$ denote the multiplication of $B$. For any non-zero elements $y \in B_{h}, z \in B_{l}, y * z=y \tau_{h}(z) \neq 0$. Hence $B$ has no homogeneous zero divisors. By [13, Proposition A.II.1.4] (the same result holds for an ordered semigroup), $B$ is a domain. Therefore being a domain is $\mathbb{G}$-twisting invariant.

By the above proof, $A^{\tau}$ is a graded domain if and only if $A$ is. Similarly we have the following. 
Proposition 5.3.1. 1. Let $\tau=\left\{\tau_{g} \mid g \in \mathbb{G}\right\}$ be a twisting system of $A$ with $\tau_{e}(1)=1$. Then a homogeneous element $a$ is invertible in $A^{\tau}$ if and only if it is invertible in $A$.

2. The ring $A^{\tau}$ is a graded division ring if and only if $A$ is.

Proof. By Proposition 2.5(2), $A$ is a twisted algebra of $A^{\tau}$. Then we only need to prove one direction and we suppose that $a$ is invertible in $A$, that is, there is $b$ such that $a b=b a=1$. Let $\operatorname{deg}(a)=h$. Then $\operatorname{deg}(b)=h^{-1}$. By the definition of * and (2.1.3), we have

$$
1=\tau_{h^{-1}}^{-1}(a b)=\tau_{h^{-1}}^{-1}(a) * \tau_{h h^{-1}}^{-1}(b)=\tau_{h^{-1}}^{-1}(a) * b,
$$

and

$$
1=b a=b * \tau_{h^{-1}}^{-1}(a) .
$$

Hence $\tau_{h^{-1}}^{-1}(a)$ is invertible in $A^{\tau}$. By Proposition 2.2(1), $a$ is invertible in $A^{\tau}$. Part 2 is an easy consequence of Part 1.

Next we study the relationship between localization and twisting and generalize [3, Proposition 8.12]. Suppose that $\mathbb{G}$ is a group and that $A$ is a $\mathbb{G}$-graded algebra. Let $S$ be a set of homogeneous elements of $A$. Then $S$ is called multiplicatively closed if it is closed under multiplication, and $S$ is called a right Ore set if

(a) $a S \cap s A$ is not empty for every homogeneous element $a \in A$ and every $s \in S$, and

(b) if $s a=0$ for some homogeneous element $a \in A$ and $s \in S$, then there is an $s^{\prime} \in S$ such that $a s^{\prime}=0$.

Given a multiplicatively closed right Ore set $S, A$ is localizable over $S$ (see [13, Lemma A.I.6.1]) and the graded quotient ring is denoted by $A_{s}$. The grading of $A_{s}$ is defined by $\operatorname{deg}\left(b s^{-1}\right)=\operatorname{deg}(b)(\operatorname{deg}(s))^{-1}[13$, Proposition A.I.6.2]. Without loss of generality, we may always assume that $1 \in S$.

Proposition 5.4. Suppose that $\mathbb{G}$ is a group and that $A$ is a $\mathbb{G}$-graded algebra. Let $S$ be a multiplicatively closed right Ore set of homogeneous elements and let $\tau=\left\{\tau_{g} \mid g \in \mathbb{G}\right\}$ be a twisting system of $A$ such that $\tau_{g}(S)=S$ for all $g \in \mathbb{G}$. Then

(1) $\tau$ can be extended to a twisting system $\tau$ of $A_{S}$;

(2) $S$ is a multiplicatively closed right Ore set of $A^{\tau}$ and $\left(A^{\tau}\right)_{S}$ is a $\mathbb{G}$-graded algebra;

(3) the twisted algebra $\left(A_{S}\right)^{\tau}$ is naturally isomorphic to $\left(A^{\tau}\right)_{S}$.

Proof. (1) By Proposition 2.4, we may assume $\tau_{g}(1)=1$ for all $g \in \mathbb{G}$. With this assumption, we define a twisting system $\tau$ of $A_{S}$, which is extended from $\tau$, by

$$
\tau_{g}\left(b r^{-1}\right)=\tau_{g}(b)\left[\tau_{g h l^{-1}} \tau_{h l^{-1}}^{-1}(r)\right]^{-1}
$$

where $\operatorname{deg}(b)=h$ and $\operatorname{deg}(r)=l$. If $r=1$, then $z=\tau$. It is easy to see that $\tau_{e}$ is the identity map. We need to check the following:

(a) $\tilde{I}_{g}$ is well-defined for every $g$,

(b) $\tau_{g}$ is $k$-linear and bijective for every $g$, and

(c) $\tau$ is a twisting system of $A_{s}$, namely it satisfies (2.1.1).

The proof of these three statements is routine and very tedious. We only prove 
(c) here and omit proofs of (a) and (b). Note that there are three other identities which are equivalent to (2.1.1). So it is enough to prove one of them. Applying $\tau^{-1}$ to $(5.4 .1)$ and replacing $b$ by $\tau_{g}^{-1}(b)$ and $r$ by $\tau_{h l^{-1}} \tau_{g h l^{-1}}^{-1}(r)$, we have

$$
z_{g}^{-1}\left(b r^{-1}\right)=\tau_{g}^{-1}(b)\left[\tau_{h l^{-1}} \tau_{g h l^{-1}}^{-1}(r)\right]^{-1} .
$$

To simplify the computation, we break the discussion into the following four cases: Case 1: $y=a$ and $z=b$; Case 2: $y=a$ and $z=r^{-1}$; Case 3: $y=s^{-1}$ and $z=b$; Case 4: $y=s^{-1}$ and $z=r^{-1}$. Here $a, b \in A$ and $r, s \in S$. The general case will follow from these four special cases. Let $\operatorname{deg}(a)=h, \operatorname{deg}(r)=i, \operatorname{deg}(b)=j$, and $\operatorname{deg}(s)=l$. Since $\tau_{g}(a)$ is an image of $\tau_{g}(a)$ for all $a \in A,(2.1 .1)$ implies that

$$
\tilde{\tau}_{g}\left(a \tau_{h}(b)\right)=\tau_{g}(a) \tilde{t}_{g h}(b)
$$

Hence (2.1.1) holds in Case 1. Let $b=1 ;(5.4 .1)$ becomes

$$
\tau_{g}\left(r^{-1}\right)=\left[\tau_{g i^{-1}} \tau_{i^{-1}}^{-1}(r)\right]^{-1},
$$

for all $r \in S$. Then the inverse function is

$$
\tau_{g}^{-1}\left(r^{-1}\right)=\left[\tau_{i^{-1}} \tau_{g i^{-1}}^{-1}(r)\right]^{-1}
$$

Combining (5.4.1), (5.4.2) and (5.4.3), we obtain

$$
\tau_{g}\left(a r^{-1}\right)=\tau_{g}(a) \tau_{g h} \tau_{h}^{-1}\left(r^{-1}\right)
$$

Thus (2.1.2) holds and so (2.1.1) holds in Case 2.

By Lemma 5.5.1 below and Case 1 and Case 2, (2.1.2) holds for $y=a$ and $z=b r^{-1}$ for all $a, b \in A$ and $r \in S$. To prove that (2.1.1) holds for Case 3, we use (2.1.3) for $y=s, z=s^{-1} b$. Note that $z$ can be written as $b^{\prime} r^{\prime-1}$ by the Ore condition. Hence we can apply (2.1.3) for $y=s, x=s^{-1} b$. We have

$$
\tau_{g l^{-1}}^{-1}(b)=\tau_{g l^{-1}}^{-1}\left(s \cdot s^{-1} b\right)=\tau_{g l^{-1}}^{-1}(s) \tau_{l} \tau_{g}^{-1}\left(s^{-1} b\right)
$$

Let $b=1 ;(5.4 .4)$ implies that

$$
1=\tilde{\tau}_{g l^{-1}}^{-1}(s) \tilde{\tau}_{l} \tilde{\tau}_{g}^{-1}\left(s^{-1}\right) \text { and }\left(\tilde{\tau}_{g l^{-1}}^{-1}(s)\right)^{-1}=\tilde{\tau}_{l} \tilde{\tau}_{g}^{-1}\left(s^{-1}\right)
$$

We re-write (5.4.4) as follows:

$$
z_{l} z_{g}^{-1}\left(s^{-1} b\right)=z_{l} \tau_{g}^{-1}\left(s^{-1}\right) z_{g l^{-1}}^{-1}(b)
$$

Let $g=e$ in $(5.4 .5)$; we have

$$
\tau_{l}\left(s^{-1} b\right)=\tau_{l}\left(s^{-1}\right) \tau_{l^{-1}}^{-1}(b) .
$$

Replacing $b$ by $\tau_{l^{-1}} \tau_{g l^{-1}}^{-1}(b)$ and $s^{-1}$ by $\tau_{g}^{-1}\left(s^{-1}\right)$ in (5.4.6), we obtain

$$
\tau_{l}\left(\tau_{g}^{-1}\left(s^{-1}\right) \tau_{l^{-1}} \tau_{g l^{-1}}^{-1}(b)\right)=\tau_{l} \tilde{\tau}_{g}^{-1}\left(s^{-1}\right) z_{g l^{-1}}^{-1}(b) .
$$


Now (5.4.5) and (5.4.7) imply that (2.1.3) holds for $y=s^{-1}$ and $z=b$. This is Case 3. Let $s, r \in S$; we have

$$
\begin{aligned}
\tau_{g}\left(s^{-1} r^{-1}\right) & =\tau_{g}\left((r s)^{-1}\right) \\
& =\left[\tau_{g(i)^{-1}} \tau_{(i)^{-1}}^{-1}(r s)\right]^{-1} \\
& =\left\{\tau_{g(i l)^{-1}}\left[\tau_{(i)^{-1}}^{-1}(r) \tau_{i} \tau_{l^{-1}}^{-1}(s)\right]\right\}^{-1} \\
& =\left[\tau_{g(i)^{-1}} \tau_{(i)^{-1}}^{-1}(r) \cdot \tau_{g l^{-1}} \tau_{i}^{-1} \tau_{i} \tau_{l^{-1}}^{-1}(s)\right]^{-1} \\
& =\left[\tau_{g(i)^{-1}} \tau_{(i l)}^{-1}(r) \cdot \tau_{g^{-1}} \tau_{l^{-1}}^{-1}(s)\right]^{-1} \\
& =\left[\tau_{g^{-1}} \tau_{l^{-1}}^{-1}(s)\right]^{-1}\left[\tau_{g(i)^{-1}} \tau_{(i l)^{-1}}^{-1}(r)\right]^{-1} \\
& =\tau_{g}\left(s^{-1}\right) \tau_{g^{-1}} \tau_{l^{-1}}^{-1}\left(r^{-1}\right) .
\end{aligned}
$$

Hence (2.1.2) holds for Case 4. Since every element $b r^{-1}$ can be written as $r^{\prime-1} b^{\prime}$, by Lemma 5.5.1 below, and Case 3 and Case 4, (2.1.1) holds for $y=s^{-1}$ and $z=b r^{-1}$. Finally by Lemma 5.5.2 below, (2.1.1) holds for all $y=a s^{-1}$ and $z=b r^{-1}$. Therefore $\tau$ is a twisting system of $A_{s}$. The twisted algebra is denoted by $\left(A_{s}\right)^{\tilde{z}}$.

(2) Let $s$ be any element in $S$ with $\operatorname{deg}(s)=l$. Since $\tau_{l}(S)=S$ and $\tau_{l}$ is bijective, $s * S=s \tau_{l}(S)=s S \subseteq S$. Hence $S$ is multiplicatively closed. Let $a$ be a homogeneous element in $A$ with $\operatorname{deg}(a)=h$. Then

$$
a * S \cap s * A=a \tau_{h}(S) \cap s \tau_{l}(A)=a S \cap s A
$$

is not empty. If $s * a=0$, then $s \tau_{l}(a)=0$. Hence there is $s^{\prime} \in S$ such that $\tau_{l}(a) s^{\prime}=0$. Applying $\tau_{l}^{-1}$, we obtain that there is $s^{\prime \prime} \in S$ such that $a * s^{\prime \prime}=0$. Therefore $S$ is a right Ore set of $A^{\tau}$. We denote the localization by $\left(A^{\tau}\right)_{s}$. By [13, Proposition A.I.6.2], $\left(A^{\tau}\right)_{S}$ is a $\mathbb{G}$-graded algebra.

(3) The map $f$ : $a \mapsto a 1^{-1}$ is a ring homomorphism from $A$ to $A_{s}$. Since $\tau$ is an extension of $\tau, f$ is also an algebra homomorphism from $A^{\tau}$ to $\left(A_{S}\right)^{z}$. Every element in $S$ is invertible in $A_{S}$, so, by Proposition 5.3, it is invertible in $\left(A_{S}\right)^{z}$. For a typical homogeneous element $a s^{-1}$ in $A_{S}=\left(A_{S}\right)^{z}$, by (5.4.3), we have

$$
a s^{-1}=a * z_{h}^{-1}\left(s^{-1}\right)=a * r^{-1}
$$

for some $r \in S$. If $a * s^{-1}=0$, then $a \tau_{h}\left(s^{-1}\right)=0$. Hence there is an $s^{\prime} \in S$, such that $a s^{\prime}=0$ and $a * \tau_{h}^{-1}\left(s^{\prime}\right)=0$. Therefore $\left(A_{S}\right)^{\tilde{\tau}}$ is the localization of $A^{\tau}$ over $S$. Thus $\left(A_{S}\right)^{\tilde{\tau}} \cong\left(A^{\tau}\right)_{S}$.

The following lemma is needed in the proof of Proposition 5.4.

LEMma 5.5. Let $T_{1}, T_{2}$ and $T_{3}$ be three multiplicatively closed subsets of homogeneous elements of $A$ such that $\tau_{g}\left(T_{i}\right)=T_{i}$ for all $g$ and $i=1,2,3$.

1. Suppose that (2.1.1) holds for $y, z \in T_{1}$, and for $y \in T_{1}, z \in T_{2}$. Then (2.1.1) holds for all $y \in T_{1}, z \in T_{1} T_{2}$.

2. Suppose that (2.1.1) holds for $y \in T_{1}, z \in T_{2} T_{3}$, for $y \in T_{1}, z \in T_{2}$, and for $y \in T_{2}, z \in T_{3}$. Then (2.1.1) holds for $y \in T_{1} T_{2}, z \in T_{3}$. 
Proof. 1. Let $a, b \in T_{1}$ and $r \in T_{2}$ and let $\operatorname{deg}(a)=h$ and $\operatorname{deg}(b)=j$. By using (2.1.1) and the equivalent identities, we have

$$
\begin{aligned}
\tau_{g}(a) \tau_{g h} \tau_{h}^{-1}(b r) & =\tau_{g}(a) \tau_{g h}\left[\tau_{h}^{-1}(b) \tau_{j} \tau_{h j}^{-1}(r)\right] \\
& =\tau_{g}(a) \tau_{g h} \tau_{h}^{-1}(b) \tau_{g h j} \tau_{h j}^{-1}(r) \\
& =\tau_{g}(a b) \tau_{g h j} \tau_{h j}^{-1}(r) \\
& =\tau_{g}(a b r) .
\end{aligned}
$$

Hence (2.1.2) (and (2.1.1)) holds for $y=a$ and $z=b r$.

The proof of Part 2 is similar and is omitted.

Proposition 5.6. Suppose that $\tau=\left\{\tau_{g} \mid g \in \mathbb{G}\right\}$ is an algebraic twisting system of $A$ such that the image semigroup $\mathrm{H}$ in $\operatorname{Aut}(A)$ of the map $g \mapsto \tau_{g}$ is a finite abelian group. Assume $A$ is a $P I$ ring. If either

(a) $A^{r}$ has no $|\mathrm{H}|$-torsion or

(b) $A^{\tau}$ has no nilpotent elements,

then the twisted algebra $A^{\tau}$ is a PI ring.

Proof. Note that a finite subsemigroup of a group is automatically a group. For every $y \in A_{h}, z \in A_{l}$ and for every $\tau_{g}$,

$$
\tau_{g}(y * z)=\tau_{g}\left(y \tau_{h}(z)\right)=\tau_{g}(y) \tau_{g h}(z)=\tau_{g}(y) \tau_{h}\left(\tau_{g}(z)\right)=\tau_{g}(y) * \tau_{g}(z) .
$$

Hence each $\tau_{g}$ is a graded algebra automorphism of $A^{\tau}$, so $\mathrm{H}$ is also a subgroup of $\operatorname{Aut}\left(A^{\tau}\right)$. Let $A^{\mathrm{H}}$ denote the set of all invariant elements $\left\{a \in A \mid \tau_{g}(a)=a\right.$ for all $g \in \mathbb{G}\}$. Since $A=A^{\tau}$ as a set, we have $A^{\mathrm{H}}=\left(A^{\tau}\right)^{\mathrm{H}}$. Both $A^{\mathrm{H}}$ and $\left(A^{\tau}\right)^{\mathrm{H}}$ are also graded algebras. For every $y \in A_{g} \cap A^{\mathrm{H}}, z \in A_{h} \cap A^{\mathrm{H}}$,

$$
y * z=y \tau_{g}(z)=y z \text {. }
$$

Therefore $A^{\mathrm{H}}=\left(A^{\tau}\right)^{\mathrm{H}}$ as a graded algebra. By assumption, $A$ is a PI ring; then the subring $A^{\mathrm{H}}$ is a PI ring and so is $\left(A^{\tau}\right)^{\mathrm{H}}$. If (a) holds, by [10, Theorem 6.5], and if (b) holds, by [10, Theorem 6.8], $A^{\tau}$ is a PI ring.

As a consequence of Proposition 5.6, if $A$ is a $\mathbb{Z}$-graded commutative domain and $f$ is a graded automorphism of $A$ of finite order, then $A^{\tau}$ is a PI ring where $\tau=\left\{f^{n} \mid n \in \mathbb{Z}\right\}$.

In the rest of this section we concentrate on connected graded algebras. Let us start with free algebras and their graded factor rings. Let $k\langle m\rangle$ denote the free algebra $k\left\langle x_{1}, \ldots, x_{m}\right\rangle$ generated by $x_{1}, \ldots, x_{m}$. Given $m$ integers $l_{1}, \ldots, l_{m} \in \mathbb{Z}$, we may assign the grading of $k\langle m\rangle$ by setting $\operatorname{deg}\left(x_{i}\right)=l_{i}$ for all $i=1, \ldots, m$. Then the free algebra $k\langle m\rangle$ is $\mathbb{Z}$-graded with $\operatorname{deg}\left(x_{i_{1}}, \ldots, x_{i_{i}}\right)=\sum_{s=1}^{t} l_{i_{s}}$. Every finitely generated $\mathbb{Z}$-graded $k$-algebra is a graded factor ring of a free algebra $k\langle m\rangle$. If $A$ is connected graded, then $A$ is isomorphic to a graded factor ring $k\langle m\rangle /(I)$ where the grading is given by $\operatorname{deg}\left(x_{i}\right)=l_{i}>0$. Let

$$
\cdots \rightarrow \bigoplus A\left[-s_{i}^{\nu}\right] \rightarrow \cdots \rightarrow \bigoplus A\left[-s_{i}^{2}\right] \rightarrow \bigoplus A\left[-s_{i}^{1}\right] \rightarrow A \rightarrow k_{A} \rightarrow 0
$$

be a minimal graded projective resolution of the trivial module $k_{A}=A / A_{\geqslant 1}$. Then the rank of the free module $\bigoplus A\left[-s_{i}^{1}\right]$ is the number of generators of $A$ and the rank of the free module $\bigoplus A\left[-s_{i}^{2}\right]$ is the number of relations of $A$. Let $B$ be a twisted algebra of $A$. Applying the equivalence functor $F$ defined in Theorem 3.4 
to (5.6.1), we obtain a minimal graded projective resolution of the trivial module $k_{B}$,

$$
\cdots \rightarrow \bigoplus B\left[-s_{i}^{\nu}\right] \rightarrow \cdots \oplus B\left[-s_{i}^{2}\right] \rightarrow \oplus B\left[-s_{i}^{1}\right] \rightarrow B \rightarrow k_{B} \rightarrow 0 .
$$

The resolutions (5.6.1) and (5.6.2) show that $k_{A}$ and $k_{B}$ have the same type of minimal graded projective resolutions. Hence $A$ and $B$ have the same number of generators and the same number of relations. Moreover, $A$ and $B$ have the same set of generators. Another immediate corollary is that $k_{A}$ and $k_{B}$ have the same projective dimension. Since $A$ is a connected graded algebra, the right global dimension of $A$ is equal to the projective dimension of $k_{A}$. This implies that $A$ and $B$ have the same right global dimension. By definition, $A$ and a twisted algebra $B$ have the same underlying graded $k$-vector space. Hence $\operatorname{dim}_{k}\left(A_{n}\right)=$ $\operatorname{dim}_{k}\left(B_{n}\right)$ for all $n \in \mathbb{N}$. The Hilbert function of $A$ is defined to be $h_{A}(n)=$ $\operatorname{dim}_{k}\left(A_{n}\right)$ for all $n \in \mathbb{N}$. Hence $A$ and $B$ have the same Hilbert function. If $A$ is a finitely generated algebra, then the Gelfand-Kirillov dimension (GK-dimension) of $A$ is equal to the number $\overline{\lim }_{N \rightarrow \infty}\left(\log \left(\sum_{n \leqslant N} h_{A}(n)\right) / \log N\right)$. Therefore $A$ and $B$ have the same GK-dimension. Parts (a) and (b) of the following proposition are proved.

Proposition 5.7. The following properties are $\mathbb{Z}$-twisting invariant for finitely generated connected graded algebras:

(a) having $G K$-dimension $d_{1}$;

(b) having right (left) global dimension $d_{2}$;

(c) having right (left) Krull dimension $d_{3}$;

(d) having right (left) uniform Goldie dimension $d_{4}$.

Proof. By Theorem 4.3, a twisted algebra is isomorphic to an l-twisted algebra. Hence we only need to consider right dimensions and it remains to prove (c) and (d). Suppose $B$ is a twisted algebra of $A$. By Theorem 3.1, there is an equivalence functor from $\mathrm{Gr}-A$ to $\mathrm{Gr}-B$ such that $F\left(A_{A}\right)=B_{B}$. Hence the graded Krull dimension and graded uniform dimension of $A_{A}$ are equal to the graded Krull dimension and graded uniform dimension of $B_{B}$, respectively. By [13, Lemma A.II.5.7], a graded Krull dimension is equal to an ungraded Krull dimension and by [13, Theorem A.II.5.10], a graded uniform dimension is equal to an ungraded uniform dimension. Hence $A$ and $B$ have the same right Krull and uniform dimensions.

A graded aigebra $A$ is called graded Goldie if $A$ has finite graded right and left uniform (Goldie) dimensions and satisfies the ascending chain condition on graded right (as well as left) annihilators. Let $A$ be a semiprime graded Goldie, connected graded algebra. By [13, Theorem C.I.1.6], $A$ has a graded semisimple, graded artinian graded quotient ring which is denoted by $Q_{\mathrm{gr}}(A)$. By the proof of [13, Theorem C.I.1.6], a connected graded algebra $A$ is semiprime graded Goldie if and only if any graded essential right (and left) ideal of $A$ contains a regular homogeneous element. It is easy to see that a graded essential right (and left) ideal and a regular homogeneous element are preserved under twisting. Therefore being semiprime graded Goldie is $\mathbb{Z}$-twisting invariant. By Proposition 5.4, we have proved the following. 
Proposition 5.8. Being semiprime graded Goldie is a $\mathbb{Z}$-twisting invariant property for connected graded algebras. Moreover, if $B$ is a twisted algebra of a semiprime graded Goldie algebra $A$, then $Q_{\mathrm{gr}}(B)$ is a twisted algebra of $Q_{\mathrm{gr}}(A)$.

We have an example which shows that being prime is not $\mathbb{Z}$-twisting invariant. It is easy to see from the definition that being graded semisimple graded artinian is $\mathbb{Z}$-twisting invariant. However, being graded simple graded artinian is not.

ExAmple 5.9. Let $A$ be the graded $k$-algebra $k\langle x, y\rangle /(x y, y x)$ with $\operatorname{deg}(x)=$ $\operatorname{deg}(y)=1$. Since $A$ is commutative and $x y=0, A$ is not prime. Let $\sigma$ be the graded algebra automorphism sending $x$ to $y$ and $y$ to $x$. The twisted algebra associated to $\left\{\tau_{n}=\sigma^{n} \mid n \in \mathbb{Z}\right\}$ is isomorphic to $B=k\langle x, y\rangle /\left(x^{2}, y^{2}\right)$. The algebra $B$ is a noetherian prime PI ring. Therefore being prime is not $\mathbb{Z}$-twisting invariant (even for connected graded algebras). By Proposition 5.8, $Q_{\mathrm{gr}}(B)$ is a twisted algebra of $Q_{\mathrm{gr}}(A)$. Since $A$ is semiprime (but not prime), $Q_{\mathrm{gr}}(A)$ is graded semisimple (but not graded simple) graded artinian. Since $B$ is prime, $Q_{\mathrm{gr}}(B)$ is graded simple graded artinian. Thus being graded simple graded artinian is not $\mathbb{Z}$-twisting invariant.

In the next example we show that being a simple ring is not $\mathbb{Z}$-twisting invariant.

ExAmPle 5.10. Let $A$ be the commutative graded $k$-algebra $k\left[x, y, x^{-1}, y^{-1}\right]$ with $\operatorname{deg}(x)=\operatorname{deg}(y)=1$. Let $\sigma$ be a graded algebra automorphism of $A$ sending $x$ to $x$ and $y$ to $q y$ where the non-zero scalar $q$ is not a root of 1 . Then the twisted algebra associated to $\sigma$ is isomorphic to $B=k_{q}\left[x, y, x^{-1}, y^{-1}\right]$ with the relation $y x=q x y$. By [11, Proposition 1.3], $B$ is simple. However, the commutative ring $A$ is not simple because it is not a field. Therefore being simple is not $\mathbb{Z}$-twisting invariant.

Let $A$ be a noetherian connected graded $k$-algebra. Recall that, for graded right $A$-modules $M$ and $N, \underline{\operatorname{Hom}}_{A}(M, N)=\bigoplus_{n \in \mathbb{Z}} \operatorname{Hom}_{\mathrm{Gr}-A}(M, N[n])$ and the derived functors of $\underline{\operatorname{Hom}}_{A}(M, N)$ are $\underline{\operatorname{Ext}}_{A}^{i}(M, N)=\bigoplus_{n \in \mathbb{Z}} \operatorname{Ext}_{\mathrm{Gr}-A}^{i}(M, N[n])$. If $M$ is a finitely generated graded right $A$-module, then $\operatorname{Hom}_{A}(M, N)$ coincides with the usual $\operatorname{Hom}_{A}(M, N)$ and $\operatorname{Ext}_{A}^{i}(M, N)$ coincides with the usual $\operatorname{Ext}_{A}^{i}(M, N)$. If $M$ and $N$ are graded left $A$-modules, $\underline{\operatorname{Hom}}_{A}(M, N)$ and $\underline{\operatorname{Ext}}_{A}^{i}(M, N)$ are defined similarly. For every graded right (or left) $A$-module $M$ and for every $i \geqslant 0$, $\operatorname{Ext}_{A}^{i}(M, A)$ is a graded left (or right) $A$-module. The grade, or $j$ number, of $M$ is defined to be $j(M)=\min \left\{j \mid \underline{\operatorname{Ext}}_{A}^{j}(M, A) \neq 0\right\}$. The GK-dimension of $M$ is denoted by $\mathrm{GKdim}(M)$, the injective dimension of $A$ is denoted by $\operatorname{inj} \operatorname{dim}(A)$, and the global dimension of $A$ is denoted by $\operatorname{gl} \operatorname{dim}(A)$. An algebra $A$ is called Cohen-Macaulay, if, for every finitely generated graded $A$-module $M$,

$$
j(M)+\mathrm{GKdim}(M)=\mathrm{GKdim}(A)<\infty .
$$

Let $A$ be a noetherian connected graded algebra with finite injective dimension. Then $A$ is called Artin-Schelter Gorenstein if $\operatorname{GKdim}(A)<\infty$ and $j\left(k_{A}\right)=$ $\operatorname{inj} \operatorname{dim}(A)$. If, moreover, $A$ has finite global dimension, then $A$ is called Artin-Schelter regular. An algebra $A$ is called Auslander Gorenstein if, for every finitely generated graded $A$-module $M$ and every submodule $N \subset \underline{\operatorname{Ext}}_{A}^{i}(M, A)$, 
$j(N) \geqslant i$. If, moreover, $A$ has finite global dimension, then $A$ is called Auslander regular. Ungraded versions of these definitions can be made by considering ungraded modules. A noetherian graded ring $A$ is Auslander Gorenstein (or regular) if and only if $A$ is graded Auslander Gorenstein (or regular) and if $A$ is Auslander Gorenstein and graded Cohen-Macaulay then $A$ is Cohen-Macaulay (see [9, Theorem 3.1 and Lemma 5.8]). Some basic facts about non-commutative graded regular rings can be found in [2] and [9].

THEOREM 5.11. The following properties are $\mathbb{Z}$-twisting invariant for noetherian connected graded $k$-algebras:

(a) having right (left) injective dimension d;

(b) being Artin-Schelter Gorenstein (or Artin-Schelter regular);

(c) being graded Cohen-Macaulay;

(d) being Auslander Gorenstein (or Auslander regular) and Cohen-Macaulay.

Proof. Suppose that $A$ is a noetherian connected graded algebra and that $B=A^{\mathrm{T}}$ is a twisted algebra of $A$. Let $F$ be the equivalence functor from $\mathrm{Gr}-A$ to Gr- $B$. By Theorem 3.4, $F(A[n]) \cong B[n]$ for all $n \in \mathbb{Z}$. Hence we have

$$
\operatorname{Ext}_{\mathrm{Gr}-A}^{i}(M, A[n]) \cong \operatorname{Ext}_{\mathrm{Gr}-B}^{i}\left(M^{\tau}, B[n]\right)
$$

for every graded $A$-module $M$. Combining this with the definition of $\operatorname{Ext}_{A}^{i}(M, A)$, we have

$$
\underline{\operatorname{Ext}}_{A}^{i}(M, A) \cong \underline{\operatorname{Ext}}_{B}^{i}\left(M^{\tau}, B\right)
$$

as graded $k$-vector spaces. Note that also since $\mathbb{Z}$ is a group, a twisted algebra is isomorphic to an $l$-twisted algebra. Hence the above argument works for both left and right modules.

(a) By (5.11.1), $A$ and $B$ have the same graded injective dimension. But graded injective dimension is equal to injective dimension for connected graded noetherian rings [9, Lemma 3.3] and therefore injective dimension is preserved under twisting.

(b) By Proposition 5.7, $\mathrm{GKdim}(A)=\mathrm{GKdim}(B)$ and $\operatorname{gl} \cdot \operatorname{dim}(A)=\operatorname{gl} \operatorname{dim}(B)$. By (a), inj.dim $(A)=\operatorname{inj} \cdot \operatorname{dim}(B)$. Since $F\left(k_{A}\right)=k_{B}$, by (5.11.1), we obtain $j\left(k_{A}\right)=$ $j\left(k_{B}\right)$. Therefore $A$ is Artin-Schelter Gorenstein (or Artin-Schelter regular) if and only if $B$ is.

(c) By (5.11.1), $j(M)=j\left(M^{\tau}\right)$ and by the definition of twisted modules, $\operatorname{dim} M_{n}=\operatorname{dim} M_{n}^{\tau}$, which implies that $\operatorname{GKdim}(M)=\operatorname{GKdim}\left(M^{\tau}\right)$. Hence $A$ is graded Cohen-Macaulay if and only if $A^{\tau}$ is.

(d) If the algebra $A$ is Cohen-Macaulay, then it is easy to show that the condition of Auslander Gorenstein is equivalent to the condition that $\operatorname{GKdim}\left(\operatorname{Ext}_{A}^{i}(M, A)\right) \leqslant i$ for every $i$ and every finitely generated graded $A$ module $M$. By (5.11.1), this condition is preserved under twisting. Combining tinis with (c), we complete our proof.

EXAMPLE 5.12: twisted algebras of commutative polynomial rings. Let $k\left[x_{1}, \ldots, x_{m}\right]$ be the polynomial ring of $m$ variables with $\operatorname{deg}\left(x_{i}\right)=l_{i}>0$. It is an Auslander regular, Cohen-Macaulay, noetherian and connected graded domain having global dimension, GK-dimension and Krull dimension $m$. By Propositions 
5.1, 5.2, 5.7 and Theorem 5.11, every twisted algebra of $k\left[x_{1}, \ldots, x_{m}\right]$ has the same properties listed. If $m=1$, every twisted algebra is isomorphic to $k[x]$ itself. If $m=2$, all twisted algebras of $k\left[x_{1}, x_{2}\right]$ are given in Example 3.6. For $m \geqslant 3$, let $B$ be a twisted algebra of $k\left[x_{1}, \ldots, x_{m}\right]$ by a twisting system $\tau=\left\{\tau_{n} \mid n \in \mathbb{Z}\right\}$ and let $*$ denote the multiplication of $B$. By the discussion before Proposition 5.7, $\left\{x_{1}, \ldots, x_{m}\right\}$ is also a set of generators of $B$ and the relations of the algebra $B$ are

$$
x_{i} * \tau_{l_{i}}^{-1}\left(x_{j}\right)-x_{j} * \tau_{l_{j}}^{-1}\left(x_{i}\right)=x_{i} \tau_{l_{i}} \tau_{l_{i}}^{-1}\left(x_{j}\right)-x_{j} \tau_{l_{i}} \tau_{l_{j}}^{-1}\left(x_{i}\right)=x_{i} x_{j}-x_{j} x_{i}=0
$$

for all $m \geqslant i>j \geqslant 1$. Roughly speaking, $B$ is isomorphic to the algebra $k\left\langle x_{1}, \ldots, x_{m}\right\rangle /(I)$ where $I$ is the homogeneous ideal generated by elements $x_{i} \tau_{l_{i}^{-1}}^{-1}\left(x_{j}\right)-x_{j} \tau_{l_{j}}^{-1}\left(x_{i}\right)$ for all $i>j$. Here we should regard $\tau_{l_{i}}^{-1}\left(x_{j}\right)$ as an element in $B$.

For example, let $A=k\left[x_{1}, x_{2}, x_{3}\right]$ with $\operatorname{deg}\left(x_{1}\right)=\operatorname{deg}\left(x_{2}\right)=1$ and $\operatorname{deg}\left(x_{3}\right)=2$. Let $f$ be an automorphism of $A$ sending

$$
x_{1} \mapsto q x_{1}, \quad x_{2} \mapsto p x_{2} \quad \text { and } \quad x_{3} \mapsto x_{3}+a x_{1}^{2}+b x_{1} x_{2}+c x_{2}^{2}
$$

for some non-zero scalars $q, p, a, b, c \in k$. Then $\tau=\left\{f^{n} \mid n \in \mathbb{Z}\right\}$ is a twisting system and the twisted algebra $A^{\tau}$ has generators $x_{1}, x_{2}, x_{3}$ and has relations

$$
\begin{aligned}
& x_{2} * q^{-1} x_{1}=x_{1} * p^{-1} x_{2}, \\
& x_{3} * q^{-2} x_{1}=x_{1} *\left(x_{3}-a q^{-3} x_{1} * x_{1}-b q^{-1} p^{-2} x_{1} * x_{2}-c p^{-3} x_{2} * x_{2}\right), \\
& x_{3} * p^{-2} x_{2}=x_{2} *\left(x_{3}-a q^{-3} x_{1} * x_{1}-b q^{-1} p^{-2} x_{1} * x_{2}-c p^{-3} x_{2} * x_{2}\right) .
\end{aligned}
$$

To obtain the last two relations note that

$$
\begin{aligned}
f^{-1}\left(x_{3}\right) & =x_{3}-a q^{-2} x_{1}^{2}-b q^{-1} p^{-1} x_{1} x_{2}-c p^{-2} x_{2}^{2} \\
& =x_{3}-a q^{-3} x_{1} * x_{1}-b q^{-1} p^{-2} x_{1} * x_{2}-c p^{-3} x_{2} * x_{2} .
\end{aligned}
$$

A natural grading of the polynomial algebra $k\left[x_{1}, \ldots, x_{m}\right]$ is defined by $\operatorname{deg}\left(x_{i}\right)=1$ for all $i$. Given a twisting system $\left\{\tau_{n} \mid n \in \mathbb{Z}\right\}$, we define an algebra automorphism $f$ by $f\left(x_{i}\right)=\tau_{1}\left(x_{i}\right)$ for all $i$ and let $\tau^{\prime}=\left\{f^{n} \mid n \in \mathbb{Z}\right\}$. Then both $k\left[x_{1}, \ldots, x_{m}\right]^{\tau}$ and $k\left[x_{1}, \ldots, x_{m}\right]^{\tau^{\prime}}$ have generators $\left\{x, \ldots, x_{m}\right\}$ and relations $x_{i} \tau_{1}^{-1}\left(x_{j}\right)-x_{j} \tau_{1}^{-1}\left(x_{i}\right)=0$ for all $i, j$. Hence $k\left[x_{1}, \ldots, x_{m}\right]^{\tau}$ is isomorphic to $k\left[x_{1}, \ldots, x_{m}\right]^{t}$. Since the automorphism $f$ is determined by an $m \times m$ invertible matrix $\alpha=\left(a_{i j}\right)_{m \times m}$ via the equation $f\left(x_{i}\right)=\sum_{j=1}^{m} a_{i j} x_{j}$, we denote $k\left[x_{1}, \ldots, x_{m}\right]^{\tau^{\prime}}$ by $k\left[x_{1}, \ldots, x_{m}\right]^{\alpha}$ for simplicity. Of course the relations of the algebra are $x_{i}\left(\sum_{s} a_{j s}^{\prime} x_{s}\right)-x_{j}\left(\sum_{s} a_{i s}^{\prime} x_{s}\right)=0$ where $\alpha^{-1}=\left(a_{i j}^{\prime}\right)_{m \times m}$ is the inverse matrix of $\alpha$. Hence we have proved the first assertion of the following proposition.

Proposinion 5.13. 1. Every twisted algebra of $k\left[x_{1}, \ldots, x_{m}\right]$ with $\operatorname{deg}\left(x_{i}\right)=1$ is isomorphic to $k\left[x_{1}, \ldots, x_{m}\right]^{\alpha}$ for some invertible matrix $\alpha$.

2. Let $\alpha$ and $\beta$ be two $m \times m$ invertible matrices. Then $k\left[x_{1}, \ldots, x_{m}\right]^{\alpha}$ is isomorphic to $k\left[x_{1}, \ldots, x_{m}\right]^{\beta}$ as graded algebras if and only if there are un :nvertible matrix $M$ and a non-zero element $c \in k$ such that $c \alpha=M \beta M^{-1}$.

Proof. In this proof let $k[m]$ denote $k\left[x_{1}, \ldots, x_{m}\right]$. Let $\alpha=\left(a_{i j}\right)_{m \times m}$ and $\beta=\left(b_{i j}\right)_{m \times m}$ be two $m \times m$ invertible matrices such that $c \alpha=M \beta M^{-1}$ for some matrix $M=\left(m_{i j}\right)_{m \times m}$ and some non-zero element $c$. We define a map $g$ from $k[m]^{\alpha}$ to $k[m]^{\beta}$ by $g\left(x_{i}\right)=\sum_{j} m_{i j} x_{j}$ for all $i$. It suffices to check that $g$ maps the 
relations of $k[m]^{\alpha}$ to the relations of $k[m]^{\beta}$. Since $\alpha=M \beta M^{-1}$, we have $\alpha^{-1} M=c M \beta^{-1}$ and for all $i, j$,

$$
\begin{aligned}
g\left(x_{i} \sum_{l} a_{j l}^{\prime} x_{l}-x_{j} \sum_{l} a_{i l}^{\prime} x_{l}\right) & =\sum_{t, l, s} m_{i l} x_{t} a_{j l}^{\prime} m_{l s} x_{s}-\sum_{t, l, s} m_{j r} x_{t} a_{i l}^{\prime} m_{l s} x_{s} \\
& =\sum_{t, l, s}\left(m_{i t} x_{t} c m_{j l} b_{l s}^{\prime} x_{s}-m_{j l} x_{t} c m_{i l} b_{l s}^{\prime} x_{s}\right) \\
& =c \sum_{t, l, s}\left(m_{i t} m_{j l} x_{t} b_{l s}^{\prime} x_{s}-m_{j l} m_{i l} x_{t} b_{l s}^{\prime} x_{s}\right) \\
& =c \sum_{t, l} m_{i t} m_{j l}\left(x_{t} \sum_{s} b_{l s}^{\prime} x_{s}-x_{l} \sum_{s} b_{l s}^{\prime} x_{s}\right)=0 .
\end{aligned}
$$

Therefore $g$ is a graded algebra isomorphism.

Conversely, suppose that $g$ is an isomorphism from $k[m]^{\alpha}$ to $k[m]^{\beta}$, and let $M=\left(m_{i j}\right)_{m \times m}$ be the matrix such that $g\left(x_{i}\right)=\sum_{j} m_{i j} x_{j}$. In the algebra $k[m]^{\beta}$, we change the set of generators from $\left\{x_{1}, \ldots, x_{m}\right\}$ to $\left\{g\left(x_{1}\right), \ldots, g\left(x_{m}\right)\right\}$. Corresponding to this new set of generators, $k[m]^{\beta}$ becomes $k[m]^{\beta_{1}}$ where $\beta_{1}=M \beta M^{-1}$, and $g$ from $k[m]^{\alpha}$ to $k[m]^{\beta_{1}}$ becomes $g\left(x_{i}\right)=x_{i}$ for all $i$. It is easy to check that $f_{\alpha^{-1}}$ defined by $f_{\alpha^{-1}}\left(x_{i}\right)=\sum_{j=1}^{m} a_{i j}^{\prime} x_{j}$ for all $i$ is an automorphism of $k[m]^{\alpha}$. Hence $f_{\alpha^{-1}}$ is also an automorphism of $k[m]^{\beta_{1}}$ and by Proposition 2.5.3,

$$
(k[m])^{\beta_{1} \cdot \alpha^{-1}}=\left(k[m]^{\beta_{1}}\right)^{\alpha^{-1}} \cong\left(k[m]^{\alpha}\right)^{\alpha^{-1}}=k[m] .
$$

Let $\gamma=\left(c_{i j}\right)_{m \times m}$ denote the product matrix $\beta_{1} \cdot \alpha^{-1}$. Then $k[m]^{\gamma}$ is a twisted algebra of $k[m]$ and it is isomorphic to $k[m]$. The relations of $k[m]^{\gamma}$ are $x_{i}\left(\sum_{s} c_{j s}^{\prime} x_{s}\right)=x_{j}\left(\sum_{s} c_{i s}^{\prime} x_{s}\right)$ for all $i, j$. Hence the commutativity implies that $c_{i j}^{\prime}=0$ for all $i \neq j$ and $c_{i i}^{\prime}=c$ for all $i$. Therefore $\beta_{1} \alpha^{-1}=\gamma=c I_{m \times m}$, and so $c \alpha=\beta_{1}=$ $M \beta M^{-1}$.

Another natural grading $k\left[x_{1}, \ldots, x_{m}\right]$ is the $\mathbb{Z}^{m}$-grading given by $\operatorname{deg}\left(x_{i}\right)=$ $(0, \ldots, 1, \ldots, 0)$ where 1 is in the $i$ th position. To construct $\mathbb{Z}^{m}$-twisted algebras we define the $\mathbb{Z}^{m}$-graded algebra automorphisms $f_{1}, \ldots, f_{m}$ of $k\left[x_{1}, \ldots, x_{m}\right]$ by $f_{i}\left(x_{j}\right)=x_{j}$ if $i \geqslant j$ and $f_{i}\left(x_{j}\right)=p_{i j}^{-1} x_{j}$ if $i<j$. Hence $\tau=\left\{f_{1}^{n_{1}} \ldots f_{m}^{n_{m}} \mid\left(n_{1}, \ldots, n_{m}\right) \in \mathbb{Z}^{m}\right\}$ is an algebraic twisting system of $k\left[x_{1}, \ldots, x_{m}\right]$ and the relations of the twisted algebra are $p_{i j} x_{i} x_{j}=x_{j} x_{i}$ for all $i, j$, where $\left\{p_{i j} \mid 1 \leqslant i<j \leqslant n\right\}$ is a set of non-zero scalars in $k$. This algebra is known as a skew polynomial ring and denoted by $k_{p_{i j}}\left[x_{1}, \ldots, x_{m}\right]$. Conversely, every $\mathbb{Z}^{m}$-graded $k$-linear automorphism $\tau_{i}$ of $k\left[x_{1}, \ldots, x_{m}\right]$ satisfies $\tau_{i}\left(x_{j}\right)=r_{i j} x_{j}$ for some non-zero element $r_{i j} \in k$. Hence $x_{i}$ and $x_{j}$ are skew-commutative in the twisted algebra and then every $\mathbb{Z}^{m}$-twisted algebra of $k\left[x_{1}, \ldots, x_{m}\right]$ is isomorphic to a skew polynomial ring $k_{p_{i j}}\left[x_{1}, \ldots, x_{m}\right]$ for some non-zero scalars $\left\{p_{i j}\right\}$. It is also easy to check that a skew polynomial ring $k_{p_{i j}}\left[x_{1}, \ldots, x_{m}\right]$ is isomorphic to a twisted algebra of $\mathbb{Z}$-graded algebra $k\left[x_{1}, \ldots, x_{m}\right]$ if and only if there are non-zero scalars $\left\{q_{i}\right\}$ such that $p_{i j}=q_{i} q_{j}^{-1}$ for all $i, j$. Finally, we want to point out that skew polynomial rings and other twisted algebras of $k\left[x_{1}, \ldots, x_{m}\right]$ can be regarded as specializations of formal deformations of $k\left[x_{1}, \ldots, x_{m}\right]$ in the sense of [6]. 


\section{Acknowledgements}

The main theorem of this paper was proved when the author was at M.I.T. as a graduate student under Professor Michael Artin. The author would like to thank Professor Artin for his encouragement and many suggestions. Without his guidance, this paper would have been impossible. The author would also like to thank Professor Toby Stafford for many fruitful conversations and useful suggestions for this paper, and Professors Géza Bohus and Tony Giaquinto for their help.

\section{References}

1. E. ABE, Hopf algebras (Cambridge University Press, 1980).

2. M. ARTIN and W. SCHELTER, 'Graded algebras of dimension 3', Adv. in Math. 66 (1987) 172-216.

3. M. ARtin, J. TATE, and M. VAN Den Bergh, 'Modules over regular algebras of dimension 3', Invent. Math. 106 (1991) 335-389.

4. M. ARTIN and J. J. ZHANG, 'Noncommutative projective schemes', Adv. in Math. 109 (1994) 228-287.

5. P. BOISEN, 'Graded Morita theory', J. Algebra 164 (1994) 1-25.

6. M. GerstenhaBer, 'On the deformation of rings and algebras', Ann. of Math. 79 (1964) 59-103.

7. R. GoRdon and E. L. GreEN, 'Graded Artin algebras', J. Algebra 76 (1980) 241-262.

8. N. JACOBSON, Basic algebra II. 2nd edn (Freeman, San Francisco, 1989).

9. T. LeVASSEUR, 'Some properties of non-commutative regular rings', Glasgow' J. Math. 34 (1992) $277-300$.

10. S. MONTGOMERY, Fixed rings of finite automorphism groups of associative rings, Lecture Notes in Mathematics 818 (Springer, Berlin, 1980).

11. J. C. MCCONnell and J. J. PetTrt, 'Crossed products and multiplicative analogues of Weyl algebras', J. London Math. Soc. (2) 38 (1988) 47-55.

12. J. C. MCCONNELL and J. C. ROBSON, Non-commutative Noetherian rings (Wiley-Interscience, Chichester, 1987).

13. C. NÄSTÁSESCL and F. VAN OYSTAEYEN, Graded ring theory (North-Holland, Amsterdam, 1982).

14. D. PASSMAN, Infinite crossed products (Academic Press, New York, 1989).

15. M. SweEdler, Hopf algebras (Benjamin, New York, 1969).

\section{University of Michigan}

Ann Arbor

Present address:

Department of Mathematics, Box 354350

University of Washington

Seattle

Washington 98195

U.S.A.

E-mail: zhang@math.washington.edu 